\title{
A New Class of Heavy-Tailed Distributions: Modeling and Simulating Actuarial Measures
}

\author{
Jin Zhao, ${ }^{1}$ Zubair Ahmad $\mathbb{D}^{2},{ }^{2}$ Eisa Mahmoudi ${ }^{\circ},{ }^{2}$ E. H. Hafez, ${ }^{3}$ and \\ Marwa M. Mohie El-Din (iD) ${ }^{4}$ \\ ${ }^{1}$ School of Finance, Shanghai Lixin University of Accounting and Finance, Shanghai, China \\ ${ }^{2}$ Department of Statistics, Yazd University, P. O. Box 89175-741, Yazd, Iran \\ ${ }^{3}$ Department of Mathematics, Faculty of Science, Helwan University, Cairo, Egypt \\ ${ }^{4}$ Department of Mathematical and Natural Sciences, Faculty of Engineering, Egyptian Russian University, Badr, Egypt
}

Correspondence should be addressed to Eisa Mahmoudi; emahmoudi@yazd.ac.ir

Received 8 February 2021; Revised 5 March 2021; Accepted 17 March 2021; Published 2 April 2021

Academic Editor: Ahmed Mostafa Khalil

Copyright ( 2021 Jin Zhao et al. This is an open access article distributed under the Creative Commons Attribution License, which permits unrestricted use, distribution, and reproduction in any medium, provided the original work is properly cited.

\begin{abstract}
Statistical distributions play a prominent role for modeling data in applied fields, particularly in actuarial, financial sciences, and risk management fields. Among the statistical distributions, the heavy-tailed distributions have proven the best choice to use for modeling heavy-tailed financial data. The actuaries are often in search of such types of distributions to provide the best description of the actuarial and financial data. This study presents a new power transformation to introduce a new family of heavy-tailed distributions useful for modeling heavy-tailed financial data. A submodel, namely, heavy-tailed beta-power transformed Weibull model is considered to demonstrate the adequacy of the proposed method. Some actuarial measures such as value at risk, tail value at risk, tail variance, and tail variance premium are calculated. A brief simulation study based on these measures is provided. Finally, an application to the insurance loss dataset is analyzed, which revealed that the proposed distribution is a superior model among the competitors and could potentially be very adequate in describing and modeling actuarial and financial data.
\end{abstract}

\section{Introduction}

Heavy-tailed distributions have been studied for decades by actuaries to investigate various aspects of financial portfolio theory and risk management problems. As always, most insurance loss datasets share the same properties such as skewed to the right, unimodal shaped, and have a very thick right tail, refer the studies of Kazemi and Noorizadeh [1], Adcock et al. [2], Bhati and Ravi [3], Ahmad et al. [4], Ahmad et al. [5], Ahmad et al. [6], and Ahmad et al. [7]. A distribution possess these characteristics is quite appropriate for modeling insurance loss data and can also be used for estimating the business risk level. These distributions include Pareto (Coorey and Ananada [8]), Lomax (Scollnik [9]), beta (Hogg and Klugman [10], lognormal (Klugman et al. [11]), Burr (Nadarajah and Bakar [12]), and Weibull (Bakar et al. [13]). These are the most appropriate models for modeling of insurance losses. For more details, refer to the studies by Shushi [14], Punzo et al. [15], Punzo et al. [16], and Punzo [17].

However, despite these classical distributions having many merits, there are still some deficiencies in these models as they are not flexible enough to provide the best fit to the heavy-tailed datasets. As an example, (i) the Pareto model, which is one of the prominent models used for modeling financial data, sometimes provides poor fitting to many financial applications, (ii) on the other hand, the Weibull model can only cover the behavior of small losses adequately but is not a reasonable candidate model to deal with the behavior of large losses, and (iii) the distribution functions of both the lognormal and the beta distributions have no closed form expressions causing difficulties in the derivation of many mathematical properties and make them less popular to use for analyzing financial datasets (Bhati and Ravi [18]). 
Due to the importance of the heavy-tailed distributions in the field of actuarial sciences, the actuaries are always in search for introducing new statistical distributions to cover the deficiencies of the traditional distributions. This motivated the researchers to search for new flexible heavy-tailed distributions.

In this context, serious efforts have been performed to propose new approaches for developing new flexible distributions such as (i) composition of two or more distributions, (ii) transformation of variables, (iii) finite mixture of distributions, and (iv) compounding of distributions. However, still these methods are suffering from some sort of deficiencies (Ahmad [19] and Ahmad et al. [20]). Thus, it is imperative to develop new statistical models having a closed form of distribution functions, heavy-tailed than the exponential class of distributions, and are capable of adequately modeling the financial risk management problems.

In the premises of the above, we further carry this area of distribution theory and propose a new method to introduce new flexible heavy-tailed distributions. The proposed family may be called a new heavy-tailed beta-power transformed (HTBPT) family of distributions. The proposed method may be an interesting alternative to the existing literature for fitting heavytailed data. We study a new model called the heavy-tailed betapower transformed Weibull (HTBPT-Weibull) distribution as a special case of the HTBPT distributions. The proposed model is very flexible and could be chosen as a good candidate model for modeling heavy-tailed data. Some mathematical properties along with the actuarial measures are derived. Based on the actuarial measures, a comparative simulation study of the proposed model and Weibull (also known as superexponential) distributions is conducted. The simulation results show that the HTBPT-Weibull distribution has heavier tail than the superexponential distribution.

The study is sectioned as follows. In Section 2, we provided the steps followed in the derivation of the proposed method. In Section 3, we introduced a special case of the proposed family. In Section 4, we introduced some mathematical properties of the proposed family. In Section 5, the maximum likelihood estimation method is used in order to estimate the parameters of the model. A brief Monte Carlo simulation study is provided in Section 6. Actuarial measures of the proposed model are discussed in Section 7. A real-life application of the proposed model to insurance loss data is provided in Section 8. Finally, Section 9 contains the conclusions and the major findings of this research study.

\section{Steps Followed for Developing the Proposed Family}

Suppose that a random variable $T \in\left[a_{1}, a_{2}\right]$ for $-\infty \leq a_{1}<a_{2}<\infty$, with density function $v(t)$, and let the random variable $X$ has the cumulative distribution function (cdf) $W[F(x ; \psi)]$, satisfying

(1) $W[F(x ; \psi)] \in\left[a_{1}, a_{2}\right]$

(2) $W[F(x ; \psi)]$ must be monotonically increasing and can be differentiated

(3) $W[F(x ; \psi)] \longrightarrow a_{1} \quad$ as $\quad x \longrightarrow-\infty \quad$ and $W[F(x ; \psi)] \longrightarrow a_{2}$ as $x \longrightarrow \infty$
The $T-X$ family method was originally proposed by Alzaatreh et al. [21] as follows:

$$
G(x)=\int_{a_{1}}^{W[F(x ; \psi)]} v(t) \mathrm{d} t, \quad x \in \mathbb{R},
$$

where $W[F(x ; \psi)]$ satisfies the above conditions. Corresponding to (1), the probability density function (pdf) is

$$
g(x)=\left\{\frac{\partial}{\partial x} W[F(x ; \psi)]\right\} v\{W[F(x ; \psi)]\}, \quad x \in \mathbb{R} .
$$

More information about the $T-X$ technique for introducing new families of distributions is given by Ahmad et al. [19]. By applying the $T-X$ technique, let us assume that $T$ follows the exponential distribution with the scale parameter, say $\lambda=1$; then, its cdf has the form given by

$$
V(t)=1-e^{-t}, \quad t \geq 0 .
$$

By differentiating equation (3) with respect to $t$, we have

$$
v(t)=e^{-t}, \quad t>0
$$

Assume that $v(t)$ is as represented in equation (4) and use $W[F(x ; \psi)]=-\log \left[1-\left\{\beta^{1-F(x ; \psi)}-\beta(1-F(x ; \psi))\right\}\right]$ in (1). Then, we have

$$
G(x ; \beta, \psi)= \begin{cases}\beta^{1-F(x ; \psi)}-\beta(1-F(x ; \psi)), & \beta>0, \beta \neq 1, x \in \mathbb{R}, \\ F(x ; \psi), & \beta=1,\end{cases}
$$

where $F(x ; \psi)$ is the cdf of the baseline model which depends on the vector parameter $\psi \in \mathbb{R}$. From expression (5), we can see that the proposed cdf reduces to the baseline cdf for $\beta=1$. The density function of equation (5) is as follows:

$$
g(x ; \beta, \psi)=f(x ; \psi)\left(\beta-(\log \beta) \beta^{1-F(x ; \psi)}\right), \quad x \in \mathbb{R} .
$$

The expression (6) can also be written as

$$
g(x ; \beta ; \psi)=\frac{\beta f(x ; \psi)}{\beta^{F(x ; \psi)}}\left\{\beta^{F(x ; \psi)}-(\log \beta)\right\}, \quad x \in \mathbb{R} .
$$

The survival function (sf) and hazard rate function (hrf) of the proposed family are given by

$$
\begin{array}{ll}
S(x ; \beta, \psi)=1-\left\{\beta^{1-F(x ; \psi)}-\beta(1-F(x ; \psi))\right\}, & x \in \mathbb{R}, \\
h(x ; \beta, \psi)=\frac{f(x ; \psi)\left\{\beta-(\log \beta) \beta^{1-F(x ; \psi)}\right\}}{1-\left\{\beta^{1-F(x ; \psi)}-\beta(1-F(x ; \psi))\right\}}, & x \in \mathbb{R},
\end{array}
$$

respectively.

The random variable $X$ with pdf (5) will be denoted by $X \sim \operatorname{HTBPT}(x ; \beta, \psi)$. For the sake of simplicity, we can omit the dependence on the parameters and simply write $G(x)=G(x ; \beta, \psi)$.

\section{Description of the Proposed Model}

This section deals with the introduction of the new HTBPT-Weibull distribution and its special cases. 
3.1. Heavy-Tailed Beta-Power Transformed Weibull Distribution. Referring to the cdf of the Weibull distribution with shape parameter $\alpha>0$ and scale parameter $\gamma>0$, $F(x ; \psi)=1-e^{-\gamma x^{\alpha}}, x>0, \alpha, \gamma>0$, and pdf $f(x ; \psi)=\alpha \gamma$ $x^{\alpha-1} e^{-\gamma x^{\alpha}}$, where $\psi=(\alpha, \gamma)$. A random variable say $X$ has the HTBPT-Weibull distribution, if its cdf is

$$
G(x ; \beta, \psi)=\beta^{e^{-\gamma x^{\alpha}}}-\beta e^{-\gamma x^{\alpha}}, \quad x \geq 0, \alpha, \beta, \gamma>0, \beta \neq 1 .
$$

The pdf, sf, and hrf of the HTBPT-Weibull distributions are given by

$$
\begin{aligned}
& g(x ; \beta, \psi)=\alpha \gamma x^{\alpha-1} e^{-\gamma x^{\alpha}}\left(\beta-(\log \beta) \beta^{e^{-\gamma x^{\alpha}}}\right), \quad x>0, \\
& S(x ; \beta, \psi)=1-\left(\beta^{e^{-\gamma x^{\alpha}}}-\beta e^{-\gamma x^{\alpha}}\right), \quad x>0, \\
& h(x ; \beta, \psi)=\frac{\alpha \gamma x^{\alpha-1} e^{-\gamma x^{\alpha}}\left(\beta-(\log \beta) \beta^{e^{-\gamma x^{\alpha}}}\right)}{1-\left(\beta^{e^{-\gamma x^{\alpha}}}-\beta e^{-\gamma x^{\alpha}}\right)}, \quad x>0 \text {, }
\end{aligned}
$$

respectively.

In order to see the effect of adding $\beta$ to the proposed distribution, we fixed the distribution's parameters ( $\alpha=1.2$ and $\gamma=1$ ) and changed the values of $\beta$ and sketched the plots. From the plots provided in Figure 1, it is clear that as the value of the additional parameter $\beta$ increases, the proposed model tends to a heavy-tailed distribution.

Plots for the cdf and sf of the proposed model are provided in Figure 2. Whereas, the plots of the hrf are shown in Figure 3.

3.2. Special Cases of the HTBPT-Weibull Distribution. This subsection deals with the subcases of the proposed distribution. Let $X$ has the HTBPT-Weibull distribution with parameters $(\alpha, \beta, \gamma)$. Then, we obtain

The Weibull distribution with parameters $\alpha$ and $\gamma$, using $\beta=1$

The one parameter Weibull distribution with parameter $\alpha$, using $\beta=\gamma=1$

The exponential distribution with parameter $\gamma$, using $\beta=\alpha=1$

The Rayleigh distribution with parameter $\gamma$, if $\beta=1$ and $\alpha=2$

The one parameter HTBPT-Weibull distribution with parameters $\alpha$ and $\beta$, if $\gamma=1$ (new)

The heavy-tailed beta-power transformed exponential distribution with parameters $\beta$ and $\gamma$, if $\alpha=1$ (new)

The HTBPTR-Rayleigh distribution with parameters $\beta$ and $\gamma$, if $\alpha=2$ (new)

\section{Mathematical Properties}

This section is devoted to some basic mathematical properties of the HTBPT family.

4.1. Shape of Density and Hazard Functions of the HTBPT Distributions. The shapes of the density function and the hrf of the HTBPT distributions can be expressed by an analytical formula. We can also find the critical points of the density function by differentiating it with respect to $x$ as follows:

$$
\frac{\partial}{\partial x} \log g(x ; \beta, \psi)=0
$$

The above equation may have more than one root. If $x=x_{0}$ is a root of the above equation, then it corresponds to (i) a local maximum, if $\left(\partial^{2} / \partial^{2} x\right) \log g(x ; \beta, \psi)<0$, (ii) a local minimum, if $\left(\partial^{2} / \partial^{2} x\right) \log g(x ; \beta, \psi)>0$, and (iii) a point of inflection, if $\left(\partial^{2} / \partial^{2} x\right) \log g(x ; \beta, \psi)=0$.

The parameter space is the same as in the HTBPT family. In a similar way, the critical points of the hazard function can be obtained by differentiation as follows:

$$
\frac{\partial}{\partial x} \log h(x ; \beta, \psi)=0 .
$$

The above equation may have more than one root. If $x=x_{o}$ is a root of the above equation, then it corresponds to (i) a local maximum, if $\left(\partial^{2} / \partial^{2} x\right) \log h(x ; \beta, \psi)<0$, (ii) a local minimum, if $\left(\partial^{2} / \partial^{2} x\right) \log h(x ; \beta, \psi)>0$, and (iii) a point of inflection, if $\left(\partial^{2} / \partial^{2} x\right) \log h(x ; \beta, \psi)=0$.

4.2. Asymptotic Behavior of HTBPT Distributions. This subsection is devoted to the asymptotic behavior of the HTBPT distributions.

As $x \longrightarrow-\infty$, we have

$$
\begin{aligned}
& G(x ; \beta, \psi) \longrightarrow 0, \\
& g(x ; \beta, \psi) \longrightarrow \beta f(x ; \psi)\{1-(\log \beta)\}, \\
& S(x ; \beta, \psi) \longrightarrow 1, \\
& h(x ; \beta, \psi) \longrightarrow \beta f(x ; \psi)\{1-(\log \beta)\} .
\end{aligned}
$$

As $x \longrightarrow \infty$, we have

$$
\begin{aligned}
& G(x ; \beta, \psi) \longrightarrow 1, \\
& g(x ; \beta, \psi) \longrightarrow f(x ; \psi)\{\beta-(\log \beta)\}, \\
& S(x ; \beta, \psi) \longrightarrow 0, \\
& h(x ; \beta, \psi) \longrightarrow \infty .
\end{aligned}
$$

4.3. Mixture Representation of the Density of HTBPT Distributions. This subsection deals with the mixture representation of the density of HTBPT distributions. We 


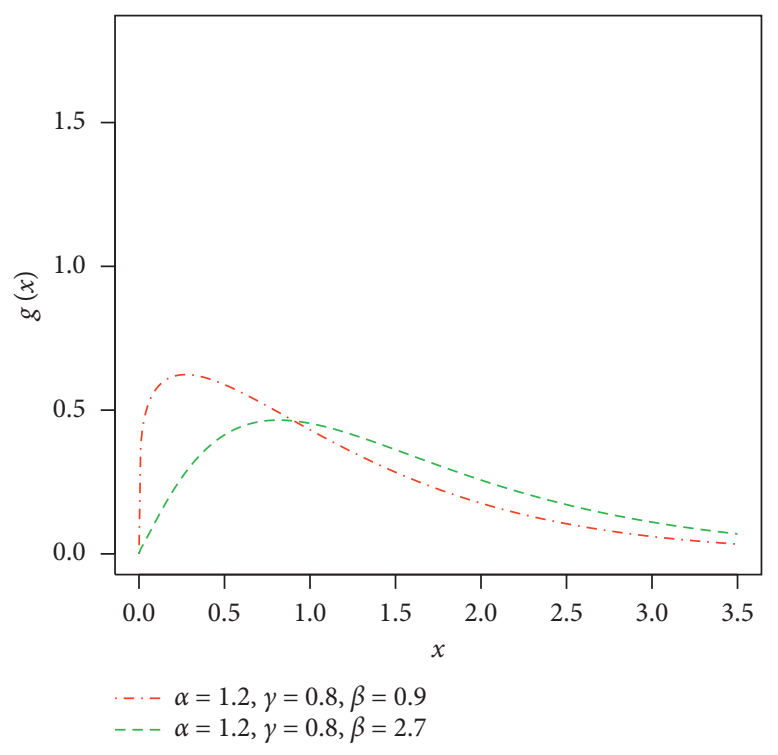

(a)

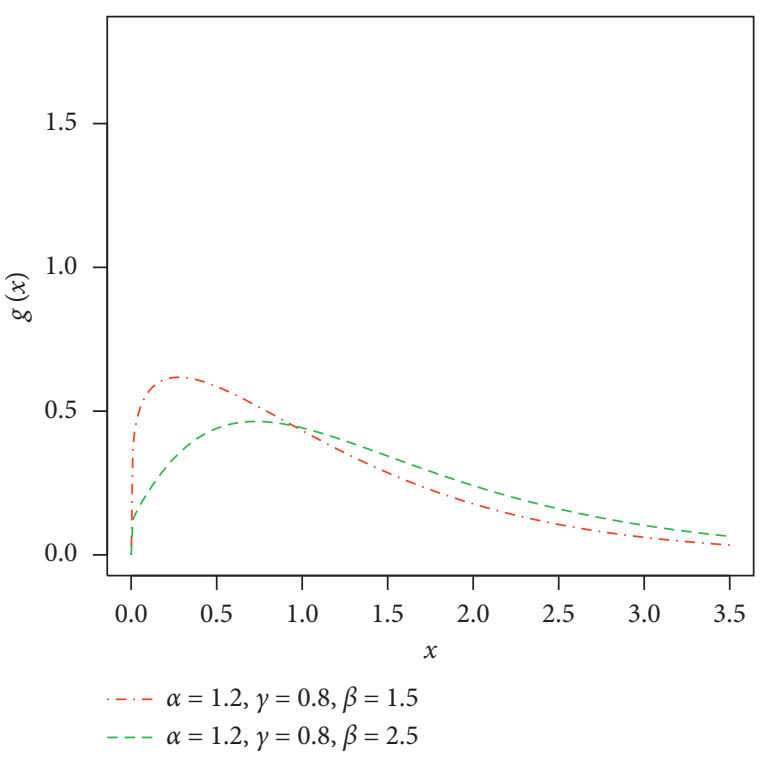

(b)

FIgURE 1: Graphical sketching of the HTBPT-Weibull density function for some parameter values.

know that $\beta^{v}=\sum_{i=0}^{\infty}\left((\log \beta)^{i} / i !\right) v^{i}$; therefore, the expression

(6) can be formulated as

$$
\begin{aligned}
& g(x ; \beta, \psi)=f(x ; \psi)\left(\beta-\sum_{i=0}^{\infty} \frac{(\log \beta)^{i+1}}{i !}(1-F(x ; \psi))^{i}\right), \\
& g(x ; \beta, \psi)=f(x ; \psi)\left(\beta-\sum_{i=0}^{\infty} \sum_{j=0}^{i} \frac{(-1)^{j}(\log \beta)^{i+1}}{i !}\left(\begin{array}{c}
i \\
j
\end{array}\right) F(x ; \psi)^{j}\right), \\
& g(x ; \beta, \psi)=\beta f(x ; \psi)-\sum_{i=0}^{\infty} \sum_{j=0}^{i} \frac{(-1)^{j}(\log \beta)^{i+1}}{i !} \frac{i !}{(i-j) ! j !} f(x ; \psi) F(x ; \psi)^{j}, \\
& g(x ; \beta, \psi)=\beta f(x ; \psi)-\sum_{i=0}^{\infty} \sum_{j=0}^{i} \frac{(-1)^{j}(\log \beta)^{i+1}}{(i-j) ! j !} f(x ; \psi) F(x ; \psi)^{j}, \\
& g(x ; \beta, \psi)=\beta f(x ; \psi)-\sum_{i=0}^{\infty} \sum_{j=0}^{i} \Lambda_{i, j} \theta f(x ; \psi) F(x ; \psi)^{\theta-1},
\end{aligned}
$$

where $\quad \Lambda_{i, j}=\left((-1)^{j}(\log \beta)^{i+1}\right) /((j+1)(i-j) ! j !), \quad$ and $j=\theta-1$.

$$
g(x ; \beta, \psi)=\beta f(x ; \psi)-\sum_{i=0}^{\infty} \sum_{j=0}^{i} \Lambda_{i, j} h_{\theta}(x ; \psi),
$$

where $h_{\theta}(x ; \psi)=\theta f(x ; \psi) F(x ; \psi)^{\theta-1}$ is defined as the density function of exponentiated generated distributions with parameter $\theta$ in its power.

4.4. Quantile Function. The quantile functions are in extensive use in general statistics and frequently used to generate random samples from probability distributions. Let
$X$ denotes the HTBPT random variable; then, the quantile function of $X$ can be obtained as follows:

$$
x=Q(u)=G^{-1}(u)=F^{-1}(t),
$$

where $t$ is the solution of the equation $\beta^{1-t}+\beta t=u+\beta$, and $u$ is the uniform distribution over $(0,1)$ interval.

4.5. Moments. In this part of the study, we derived an important property of the HTBPT distributions called moments, and it has a specific and important role in statistical analysis. It is used in finding the major properties and characteristics of the distribution (e.g., central tendency, 


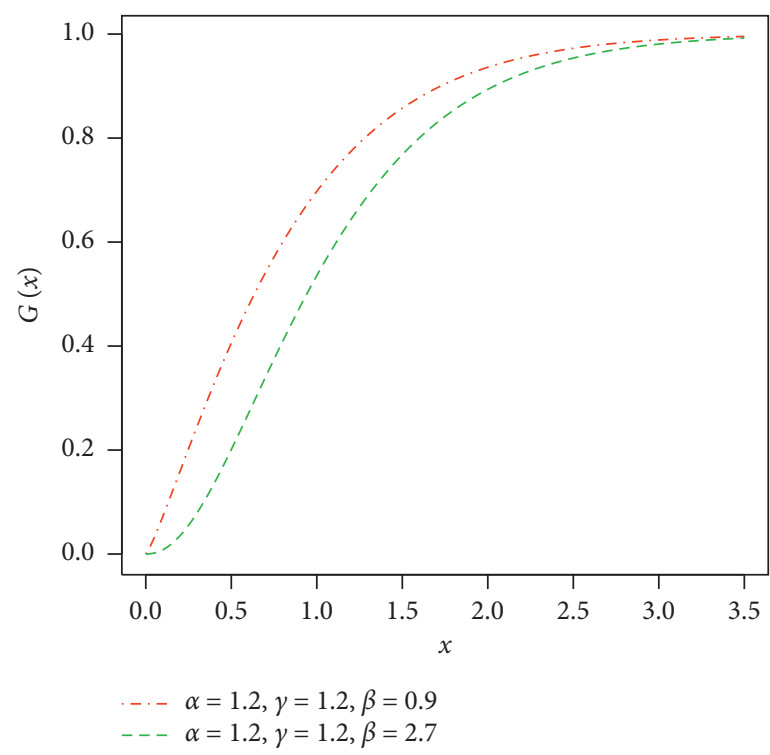

(a)

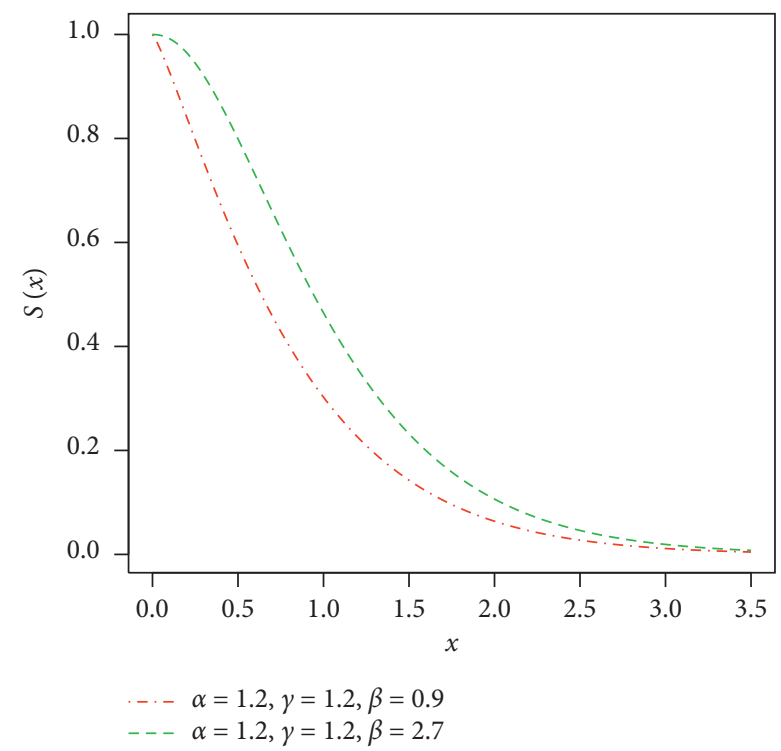

(b)

FIGURE 2: Graphical sketching of the HTBPT-Weibull cdf and sf plots for selected parameter values.

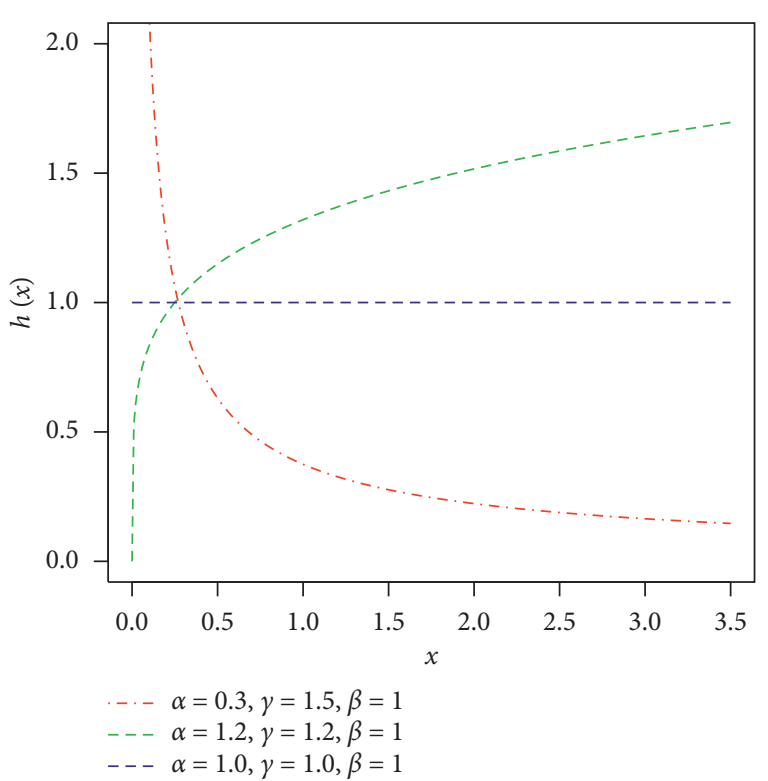

FIGURE 3: Graphical sketching of the HTBPT-Weibull hrf for some parameter values.

dispersion, skewness, and kurtosis). The $r^{\text {th }}$ moment of the HTBPT distributions is derived as follows:

$$
\begin{aligned}
\mu_{r}^{\prime} & =\int_{-\infty}^{\infty} x^{r} g(x ; \beta, \psi) \mathrm{d} x \\
& =\beta \int_{-\infty}^{\infty} x^{r} f(x ; \psi) \mathrm{d} x-\int_{-\infty}^{\infty} \sum_{i, j=0}^{\infty} \eta_{i, j} x^{r} f(x ; \psi) F(x ; \psi)^{j} \mathrm{~d} x \\
& =\beta v_{r}-\sum_{i, j=0}^{\infty} \eta_{i, j} v_{r, j},
\end{aligned}
$$

where

$$
\begin{aligned}
\eta_{i, j} & =\sum_{i=0}^{\infty} \frac{(\log \beta)^{i+1}}{i !}\left(\begin{array}{c}
i \\
j
\end{array}\right), \\
v_{r} & =\int_{-\infty}^{\infty} x^{r} f(x ; \psi) \mathrm{d} x, \\
v_{r, j} & =\int_{-\infty}^{\infty} x^{r} f(x ; \psi)[F(x ; \psi)]^{j} \mathrm{~d} x .
\end{aligned}
$$

The effects of different values of the parameters $\alpha$ and $\beta$ on the mean, variance, skewness, and kurtosis of the HTBPT-Weibull distribution with $\gamma=1$ are illustrated in Figures 4 and 5 .

The moment generating function (mgf) is another useful tool to calculate the moments of the statistical distributions. The mgf of the HTBPT distributions is given by

$$
\begin{aligned}
M_{X}(t) & =\int_{-\infty}^{\infty} e^{t x} g(x ; \zeta, \psi) \mathrm{d} x \\
& =\sum_{r=0}^{\infty} \frac{t^{r}}{r !} \mu_{r}^{\prime}=\sum_{i, r=0}^{\infty} \frac{t^{r}}{r !}\left(\beta v_{r}-\sum_{i, j=0}^{\infty} \eta_{i, j} v_{r, j}\right) .
\end{aligned}
$$

4.6. Incomplete Moments. The shape of many statistical distributions can be conveniently described by the incomplete moments. It plays a very important role in measuring inequalities (e.g., income quantiles as well as Lorenz and Bonferroni curves). Here, we drive the $r^{\text {th }}$ incomplete moment of HTBPT distributions as 


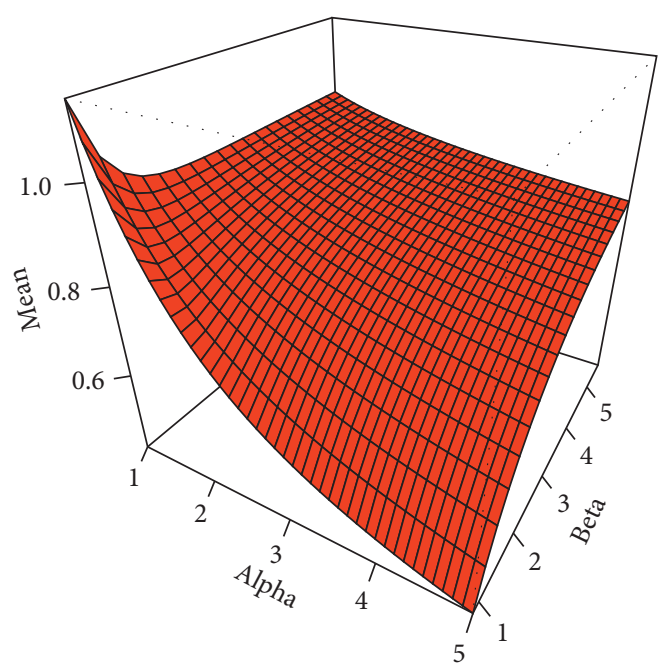

(a)

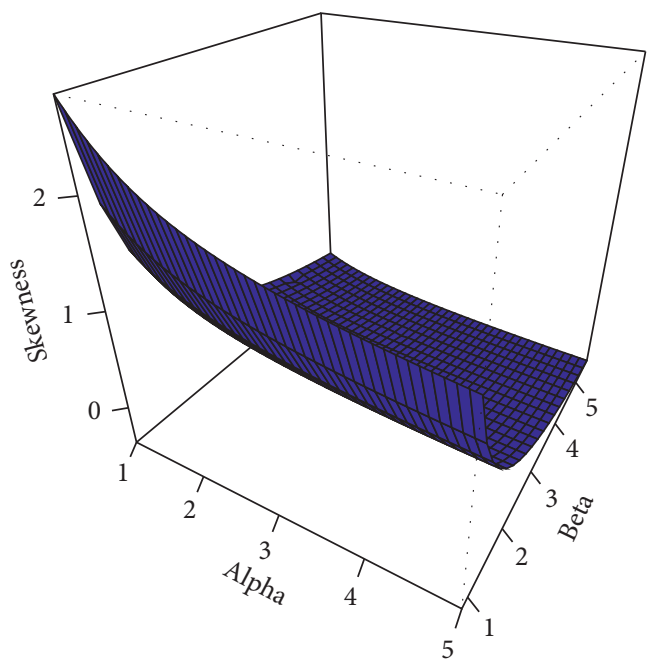

(c)

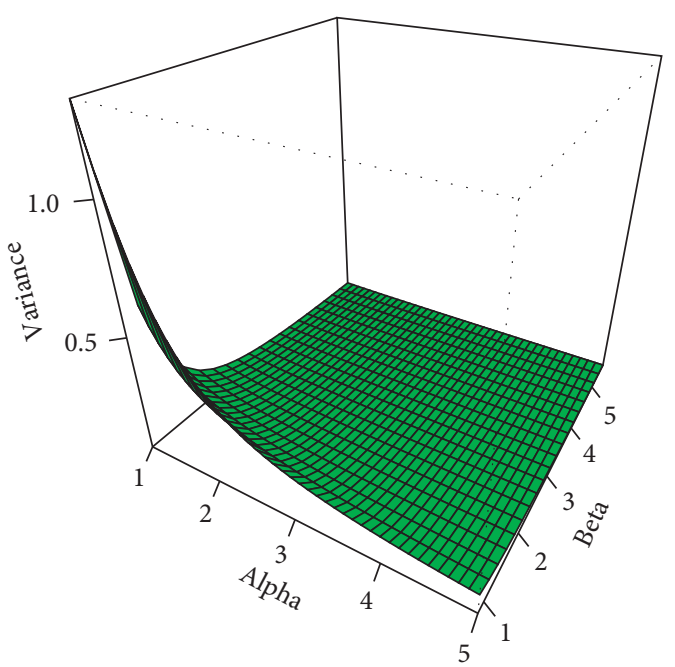

(b)

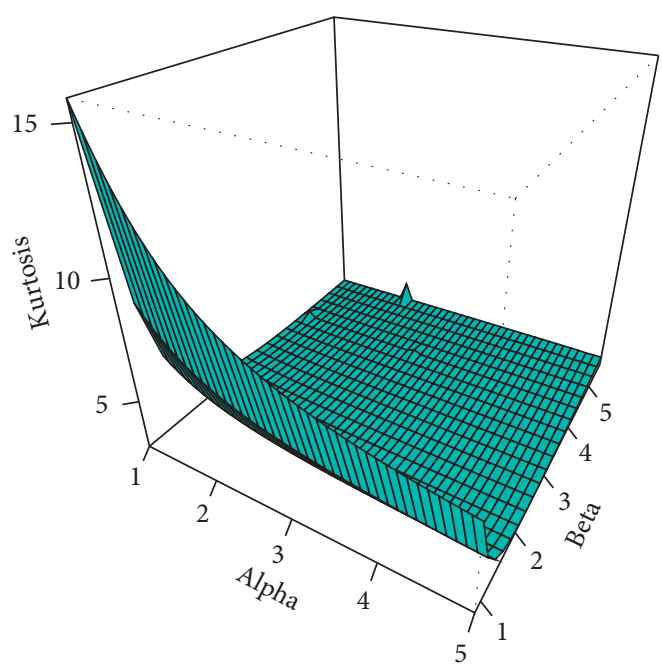

(d)

FIgURE 4: Graphical display of the mean, variance, skewness, and kurtosis of the proposed model.

$$
\begin{aligned}
\Upsilon_{r}(x) & =\int_{-\infty}^{x} x^{r} g(x ; \beta, \psi) \mathrm{d} x \\
& =\beta \int_{-\infty}^{x} x^{r} f(x ; \psi) \mathrm{d} x-\int_{-\infty}^{x} \sum_{i, j=0}^{\infty} \eta_{i, j} x^{r} f(x ; \psi)[F(x ; \psi)]^{j} \mathrm{~d} x \\
& =\beta b_{r}-\sum_{i, j=0}^{\infty} \eta_{i, j} b_{r, j},
\end{aligned}
$$

where $\quad b_{r}=\int_{-\infty}^{x} x^{r} f(x ; \psi) \mathrm{d} x$, and $\quad b_{r, j}=\int_{-\infty}^{x} x^{r} f$ $(x ; \psi) F(x ; \psi)^{j} \mathrm{~d} x$. Furthermore, we can find the conditional measures of the HTBPT-distributed random variable $X$ as

$$
\begin{aligned}
E\left(\frac{X^{r}}{X}>t\right)= & \frac{1}{S(t ; \psi)} \beta \int_{-\infty}^{\infty} x^{r} f(x ; \psi) \mathrm{d} x \\
& -\frac{1}{S(t ; \psi)} \int_{-\infty}^{\infty} \sum_{i, j=0}^{\infty} \eta_{i, j} x^{r} f(x ; \psi) F(x ; \psi)^{j} \mathrm{~d} x \\
= & \frac{1}{S(t ; \psi)}\left(\beta \Lambda_{r}-\sum_{i, j=0}^{\infty} \eta_{i, j} \Lambda_{r, j}\right),
\end{aligned}
$$




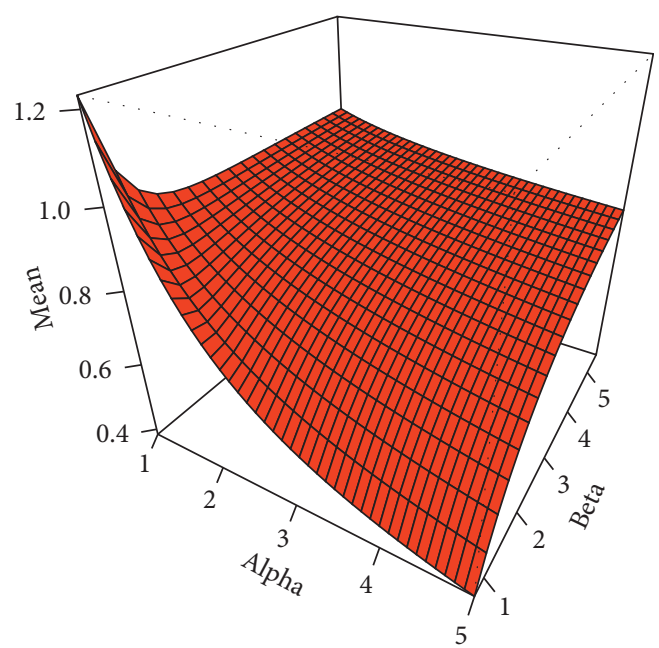

(a)

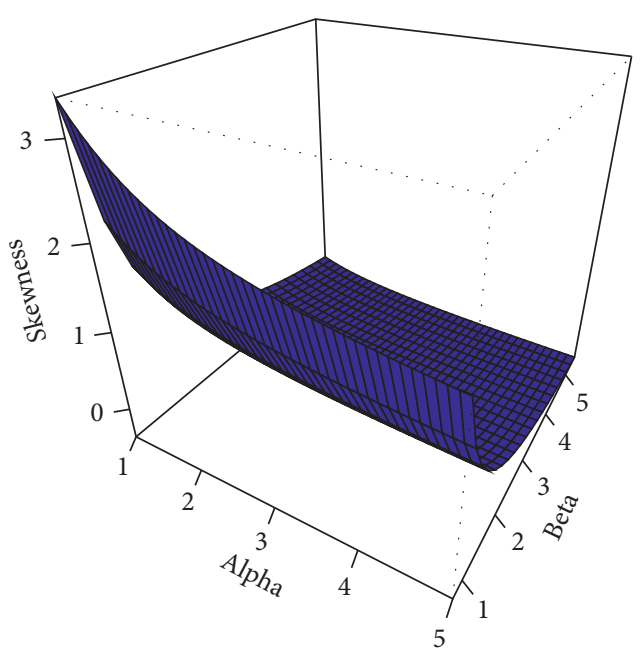

(c)

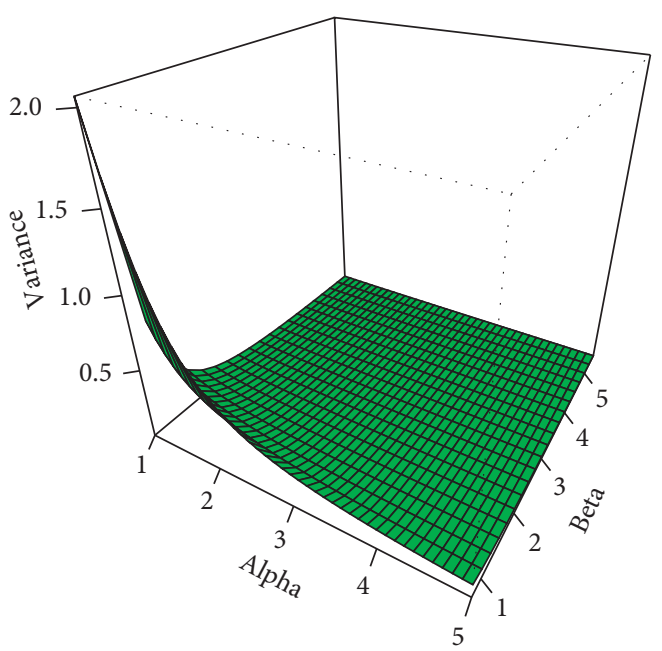

(b)

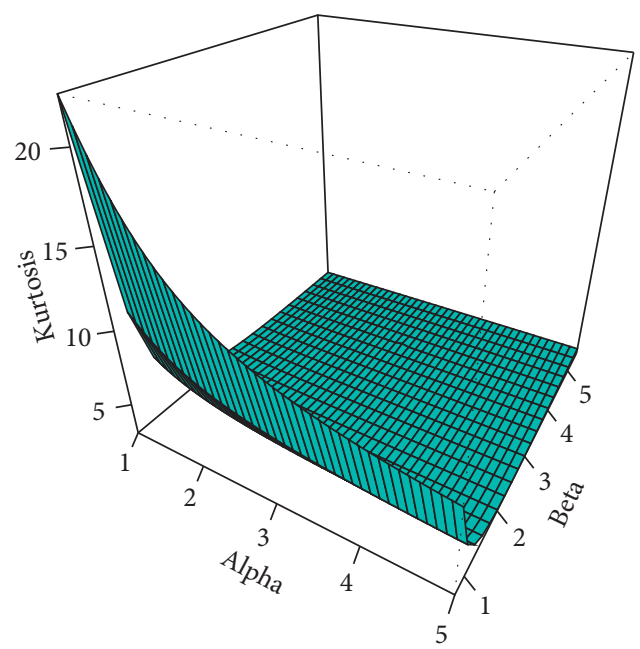

(d)

Figure 5: Graphical display of the mean, variance, skewness, and kurtosis of the proposed model.

where $\Lambda_{r}=\int_{-\infty}^{\infty} x^{r} f(x ; \psi) \mathrm{d} x$, and $\Lambda_{r, j}=\int_{-\infty}^{\infty} x^{r} f(x ; \psi)$ $[F(x ; \psi)]^{j} \mathrm{~d} x$.

\section{Estimation}

This part of the study is concerned with the estimation of the unknown parameters of the HTBPT distributions. Let $X_{1}, X_{2}, \ldots, X_{n}$ be a random sample taken from НTBPT distributions with parameters $(\beta, \psi)$. Then, the corresponding log-likelihood function of this sample is

$$
\begin{aligned}
\log L\left(x_{i} ; \beta, \psi\right)= & \sum_{i=1}^{n} \log f\left(x_{i} ; \psi\right) \\
& +\sum_{i=1}^{n} \log \left(\beta-(\log \beta) \beta^{1-F\left(x_{i} ; \psi\right)}\right) .
\end{aligned}
$$

The function (23) can be maximized either by deploying Ox program (subroutine Max BFGS) or SAS (PROC
NLMIXED) or by solving it manually. Obtaining the partial derivatives of (10), we have

$$
\begin{aligned}
\frac{\partial}{\partial \beta} L\left(x_{i} ; \beta, \psi\right)= & \sum_{i=1}^{n} \frac{1-\left(\left(1+\left\{(\log \beta)\left(1-F\left(x_{i} ; \psi\right)\right)\right\}\right) / \beta^{F\left(x_{i} ; \psi\right)}\right)}{\left(\beta-(\log \beta) \beta^{1-F\left(x_{i} ; \psi\right)}\right)}, \\
\frac{\partial}{\partial \psi} L\left(x_{i} ; \beta, \psi\right)= & \sum_{i=1}^{n} \frac{\left(\partial f\left(x_{i} ; \psi\right) / \partial \psi\right)}{f\left(x_{i} ; \psi\right)} \\
& +\sum_{i=1}^{n} \frac{(\log \beta)^{2} \beta^{1-F\left(x_{i} ; \psi\right)}\left(\partial F\left(x_{i} ; \psi\right) / \partial \psi\right)}{\left(\beta-(\log \beta) \beta^{1-F\left(x_{i} ; \psi\right)}\right)} .
\end{aligned}
$$

Equating $(\partial / \partial \beta) \log L\left(x_{i} ; \beta, \psi\right)$ and $(\partial / \partial \psi) \log L\left(x_{i} ; \beta, \psi\right)$ to zero and simultaneously solving these expressions numerically yields the maximum likelihood estimators (MLEs) of $(\beta, \psi)$. 


\section{Simulation Study}

The section offers a brief Monte Carlo simulation study to evaluate the efficiency and consistency properties of the MLEs of the HTBPT-Weibull parameters (a particular case from the proposed family). The random samples are generated from the HTBPT-Weibull distribution using the inverse cdf method. The simulation results are obtained using optim $\mathrm{R}$ function with the argument method= "LBFGS-B." The steps taken for simulation are given as follows:

(1) $N=1000$ iterations are made, and different samples of size $n=25,50, \ldots, 1000$ are generated from the HTBPT-Weibull distribution using the inversion cdf method.

(2) The parameter values are estimated using the maximum likelihood estimation method

(3) The biases and mean square errors (MSEs) are calculated using the formulas

$$
\begin{array}{r}
\operatorname{Bias}(\widehat{\alpha})=\frac{1}{1000} \sum_{i=1}^{1000}\left(\widehat{\alpha}_{i}-\alpha\right), \\
\operatorname{MSE}(\widehat{\alpha})=\frac{1}{1000} \sum_{i=1}^{1000}\left(\widehat{\alpha}_{i}-\alpha\right)^{2},
\end{array}
$$

respectively

(4) For calculating the biases as well as the MSEs of $(\beta, \gamma)$, step (3) is repeated

The simulation results are presented in Tables 1 and 2 . Whereas, the graphical display of the simulation results is shown in Figures 6-9. It is important to note that, in Figures $6-9, \zeta$ is used for $\alpha$, and $\eta$ is used for $\gamma$.

From the simulation results provided in Tables 1 and 2, it is clear that the estimates of the model parameters are very precise and close to the true value of the parameters as the samples' size increases. Also, we can see that the values of the estimated biases and absolute biases tend to zero, when the sample size $n$ increases, which shows the accuracy of MLEs. Also, as $n$ increases, MSEs tend to zero. This fact leads to the consistency property of the MLEs of the HTBPT-Weibull distribution.

\section{Actuarial Measures}

One of the primary tasks of actuarial sciences institutions is to predict the market risks in a portfolio of instruments. Henceforth, estimating the risk measures is very important in selling and buying products. In this section of the study, we discuss some important risk measures such as the value at risk (VaR), tail value at risk (TVaR), tail variance (TV), and tail variance premium (TVP) of the proposed family.

7.1. Value at Risk. The VaR of a random variable $X$ is the $q^{\text {th }}$ quantile of its cdf (Artzner [22]). If $X$ follows the HTBPT
TABLE 1: Monte Carlo simulation results of the HTBPT-Weibull

\begin{tabular}{|c|c|c|c|c|}
\hline \multicolumn{5}{|c|}{ Set 1: $\alpha=0.9, \beta=1.2, \gamma=1.5$} \\
\hline$n$ & Parameters & MLE & MSEs & Biases \\
\hline \multirow{3}{*}{25} & $\widehat{\alpha}$ & 0.735 & 0.057 & -0.164 \\
\hline & $\widehat{\beta}$ & 2.588 & 3.611 & 1.388 \\
\hline & $\hat{\gamma}$ & 2.049 & 0.552 & 0.549 \\
\hline \multirow{3}{*}{100} & $\widehat{\alpha}$ & 0.811 & 0.021 & -0.088 \\
\hline & $\widehat{\beta}$ & 1.684 & 0.571 & 0.484 \\
\hline & $\hat{\gamma}$ & 1.702 & 0.104 & 0.202 \\
\hline \multirow{3}{*}{200} & $\widehat{\alpha}$ & 0.844 & 0.010 & -0.055 \\
\hline & $\widehat{\beta}$ & 1.469 & 0.187 & 0.269 \\
\hline & $\hat{\gamma}$ & 1.622 & 0.043 & 0.122 \\
\hline \multirow{3}{*}{300} & $\widehat{\alpha}$ & 0.858 & 0.006 & -0.041 \\
\hline & $\widehat{\beta}$ & 1.397 & 0.112 & 0.197 \\
\hline & $\hat{\gamma}$ & 1.590 & 0.028 & 0.090 \\
\hline \multirow{3}{*}{400} & $\widehat{\alpha}$ & 0.866 & 0.005 & -0.033 \\
\hline & $\widehat{\beta}$ & 1.348 & 0.073 & 0.148 \\
\hline & $\hat{\gamma}$ & 1.569 & 0.019 & 0.069 \\
\hline \multirow{3}{*}{500} & $\widehat{\alpha}$ & 0.874 & 0.003 & -0.025 \\
\hline & $\widehat{\beta}$ & 1.313 & 0.052 & 0.113 \\
\hline & $\hat{\gamma}$ & 1.553 & 0.015 & 0.053 \\
\hline \multirow{3}{*}{600} & $\widehat{\alpha}$ & 0.876 & 0.003 & -0.023 \\
\hline & $\widehat{\beta}$ & 1.305 & 0.046 & 0.105 \\
\hline & $\hat{\gamma}$ & 1.552 & 0.013 & 0.052 \\
\hline \multirow{3}{*}{700} & $\widehat{\alpha}$ & 0.880 & 0.003 & -0.019 \\
\hline & $\widehat{\beta}$ & 1.289 & 0.040 & 0.089 \\
\hline & $\hat{\gamma}$ & 1.539 & 0.011 & 0.039 \\
\hline \multirow{3}{*}{800} & $\widehat{\alpha}$ & 0.882 & 0.002 & -0.017 \\
\hline & $\widehat{\beta}$ & 1.272 & 0.032 & 0.072 \\
\hline & $\hat{\gamma}$ & 1.535 & 0.009 & 0.035 \\
\hline \multirow{3}{*}{900} & $\widehat{\alpha}$ & 0.884 & 0.002 & -0.015 \\
\hline & $\widehat{\beta}$ & 1.271 & 0.029 & 0.071 \\
\hline & $\hat{\gamma}$ & 1.535 & 0.008 & 0.035 \\
\hline \multirow{3}{*}{1000} & $\widehat{\alpha}$ & 0.886 & 0.002 & -0.013 \\
\hline & $\widehat{\beta}$ & 1.260 & 0.024 & 0.060 \\
\hline & $\hat{\gamma}$ & 1.529 & 0.007 & 0.029 \\
\hline
\end{tabular}
distribution.

distributions with parameters $(\beta, \psi)$, then the $\operatorname{VaR}$ of $X$ denoted by $\operatorname{VaR}_{q}(X)$ is given by

$$
x_{q}=F^{-1}(t)
$$

where $t$ is the solution of the equation $\beta^{1-t}+\beta t=u+\beta$, and $u$ follows the uniform distribution on interval $(0,1)$.

7.2. Tail Value at Risk. Let $X$ follows the HTBPT distributions with parameters $\beta$ and $\psi$; then, the TVaR of $X$ is derived as

$$
\begin{aligned}
& \operatorname{TVaR}_{q}(X)=E\left(X \mid X>\operatorname{VaR}_{q}(X)\right) \\
& \operatorname{TVaR}_{q}(X)=\frac{1}{1-q} \int_{\operatorname{VaR}_{q}}^{\infty} x g(x ; \beta, \psi) \mathrm{d} x .
\end{aligned}
$$

Using (6) in (27), we get 
TABle 2: Monte Carlo simulation results of the HTBPT-Weibull distribution.

\begin{tabular}{|c|c|c|c|c|}
\hline \multicolumn{5}{|c|}{ Set $2: \alpha=1.2, \beta=1.5, \gamma=0.5$} \\
\hline$n$ & Parameters & MLE & MSEs & Biases \\
\hline \multirow{3}{*}{25} & $\widehat{\alpha}$ & 1.072 & 0.130 & -0.127 \\
\hline & $\widehat{\beta}$ & 2.898 & 4.418 & 1.498 \\
\hline & $\widehat{\gamma}$ & 0.756 & 0.199 & 0.256 \\
\hline \multirow{3}{*}{100} & $\widehat{\alpha}$ & 1.152 & 0.040 & -0.047 \\
\hline & $\widehat{\beta}$ & 2.019 & 1.580 & 0.619 \\
\hline & $\widehat{\gamma}$ & 0.578 & 0.041 & 0.078 \\
\hline \multirow{3}{*}{200} & $\widehat{\alpha}$ & 1.167 & 0.027 & -0.032 \\
\hline & $\widehat{\beta}$ & 1.800 & 0.994 & 0.400 \\
\hline & $\hat{\gamma}$ & 0.549 & 0.022 & 0.049 \\
\hline \multirow{3}{*}{300} & $\widehat{\alpha}$ & 1.186 & 0.013 & -0.013 \\
\hline & $\widehat{\beta}$ & 1.750 & 0.745 & 0.280 \\
\hline & $\hat{\gamma}$ & 0.526 & 0.011 & 0.026 \\
\hline \multirow{3}{*}{400} & $\widehat{\alpha}$ & 1.188 & 0.010 & -0.011 \\
\hline & $\widehat{\beta}$ & 1.714 & 0.431 & 0.174 \\
\hline & $\hat{\gamma}$ & 0.520 & 0.008 & 0.020 \\
\hline \multirow{3}{*}{500} & $\widehat{\alpha}$ & 1.196 & 0.010 & -0.003 \\
\hline & $\widehat{\beta}$ & 1.680 & 0.422 & 0.150 \\
\hline & $\hat{\gamma}$ & 0.512 & 0.007 & 0.012 \\
\hline \multirow{3}{*}{600} & $\widehat{\alpha}$ & 1.193 & 0.010 & -0.006 \\
\hline & $\widehat{\beta}$ & 1.657 & 0.351 & 0.127 \\
\hline & $\hat{\gamma}$ & 0.512 & 0.008 & 0.012 \\
\hline \multirow{3}{*}{700} & $\widehat{\alpha}$ & 1.199 & 0.006 & -0.005 \\
\hline & $\widehat{\beta}$ & 1.610 & 0.215 & 0.080 \\
\hline & $\hat{\gamma}$ & 0.506 & 0.005 & 0.010 \\
\hline \multirow{3}{*}{800} & $\widehat{\alpha}$ & 1.200 & 0.006 & 0.003 \\
\hline & $\widehat{\beta}$ & 1.573 & 0.219 & 0.073 \\
\hline & $\hat{\gamma}$ & 0.505 & 0.004 & 0.009 \\
\hline \multirow{3}{*}{900} & $\widehat{\alpha}$ & 1.201 & 0.006 & 0.001 \\
\hline & $\widehat{\beta}$ & 1.530 & 0.179 & 0.060 \\
\hline & $\hat{\gamma}$ & 0.505 & 0.004 & 0.007 \\
\hline \multirow{3}{*}{1000} & $\widehat{\alpha}$ & 1.198 & 0.004 & -0.001 \\
\hline & $\widehat{\beta}$ & 1.506 & 0.086 & 0.030 \\
\hline & $\hat{\gamma}$ & 0.506 & 0.003 & 0.006 \\
\hline
\end{tabular}

$$
\begin{aligned}
\operatorname{TVaR}_{q}(X)= & \frac{1}{1-q} \int_{\operatorname{VaR}_{q}}^{\infty} x\left\{f(x ; \psi)\left(\beta-(\log \beta) \beta^{1-F(x ; \psi)}\right)\right\} \mathrm{d} x, \\
\operatorname{TVaR}_{q}(X)= & \frac{1}{(1-q)} \beta \int_{\operatorname{VaR}_{q}}^{\infty} x f(x ; \psi) \mathrm{d} x \\
& -\frac{1}{(1-q)} \sum_{i, j=0}^{\infty} \frac{(-1)^{j}(\log \beta)^{i+1}}{i !} \\
& \cdot\left(\begin{array}{c}
i \\
j
\end{array}\right) \int_{\operatorname{VaR}_{q}}^{\infty} x f(x ; \psi) F(x ; \psi)^{j} \mathrm{~d} x .
\end{aligned}
$$

Using the pdf $f(x ; \psi)$ and $\operatorname{cdf} F(x ; \psi)$ of Weibull distribution in (28), we get

$$
\begin{aligned}
& \operatorname{TVaR}_{q}(X)=\frac{1}{(1-q)} \frac{\beta}{\eta^{1 / \alpha}} \int_{\gamma\left(\operatorname{VaR}_{q}\right)^{\alpha}}^{\infty} t^{(1 / \alpha)+1-1} e^{-t} \mathrm{~d} t \\
& -\frac{1}{(1-q)} \sum_{i=0}^{\infty} \sum_{j=0}^{i} \sum_{t=0}^{j} \frac{(-1)^{j+t}(\log \beta)^{i+1}}{i ! \gamma^{1 / \alpha}(t+1)^{(1 / \alpha)+1}}\left(\begin{array}{l}
i \\
j
\end{array}\right)\left(\begin{array}{l}
j \\
t
\end{array}\right)
\end{aligned}
$$

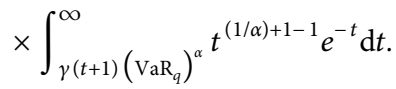

On solving (29), we have

$$
\begin{aligned}
\operatorname{TVaR}_{q}(X)= & \frac{1}{\gamma^{1 / \alpha}(1-q)} \beta \gamma\left(\frac{1}{\alpha}+1, \gamma\left(\operatorname{VaR}_{q}\right)^{\alpha}\right) \\
& -\frac{1}{\gamma^{1 / \alpha}(1-q)} \sum_{i=0}^{\infty} \sum_{j=0}^{i} \sum_{t=0}^{j} \frac{(-1)^{j+t}(\log \beta)^{i+1}}{(i-j) !(j-t) ! t !(t+1)^{(1 / \alpha)+1}} \\
& \times \gamma\left(\frac{1}{\alpha}+1, \gamma(t+1)\left(\operatorname{VaR}_{q}\right)^{\alpha}\right) .
\end{aligned}
$$

7.3. Tail Variance. Among the actuarial measures, TV is the most prominent one and deals with the tail variance beyond VaR. The TV of the HTBPT-Weibull distributed random variable is derived as follows:

$$
\mathrm{TV}_{q}(X)=E\left(X^{2} \mid X>x_{q}\right)-\left(\mathrm{TVaR}_{q}\right)^{2} .
$$

Consider

$E\left(X^{2} \mid X>x_{q}\right)=\frac{1}{1-q} \int_{\operatorname{VaR}_{q}}^{\infty} x^{2}\left\{f(x ; \psi)\left(\beta-(\log \beta) \beta^{1-F(x ; \psi)}\right)\right\} \mathrm{d} x$.

By solving the above equations, we get

$$
\begin{aligned}
E\left(X^{2} \mid X>x_{q}\right)= & \frac{1}{\gamma^{2 / \alpha}(1-q)} \beta \gamma\left(\frac{2}{\alpha}+1, \gamma\left(\operatorname{VaR}_{q}\right)^{\alpha}\right) \\
& -\frac{1}{\gamma^{2 / \alpha}(1-q)} \sum_{i=0}^{\infty} \sum_{j=0}^{i} \sum_{t=0}^{j}(-1)^{j+t}(\log \beta)^{i+1} \\
& \times \frac{\gamma\left((2 / \alpha)+1, \gamma(t+1)\left(\mathrm{VaR}_{q}\right)^{\alpha}\right)}{(i-j) !(j-t) ! t !(t+1)^{(2 / \alpha)+1}}
\end{aligned}
$$

By referring to equations (30) and (33) and substituting in (31), we get the TV of HTBPT-Weibull distribution.

7.4. Tail Variance Premium. In this subsection, we discuss another most important risk measure called TVP. The TVP of the HTBPT-Weibull distribution is derived as follows: 


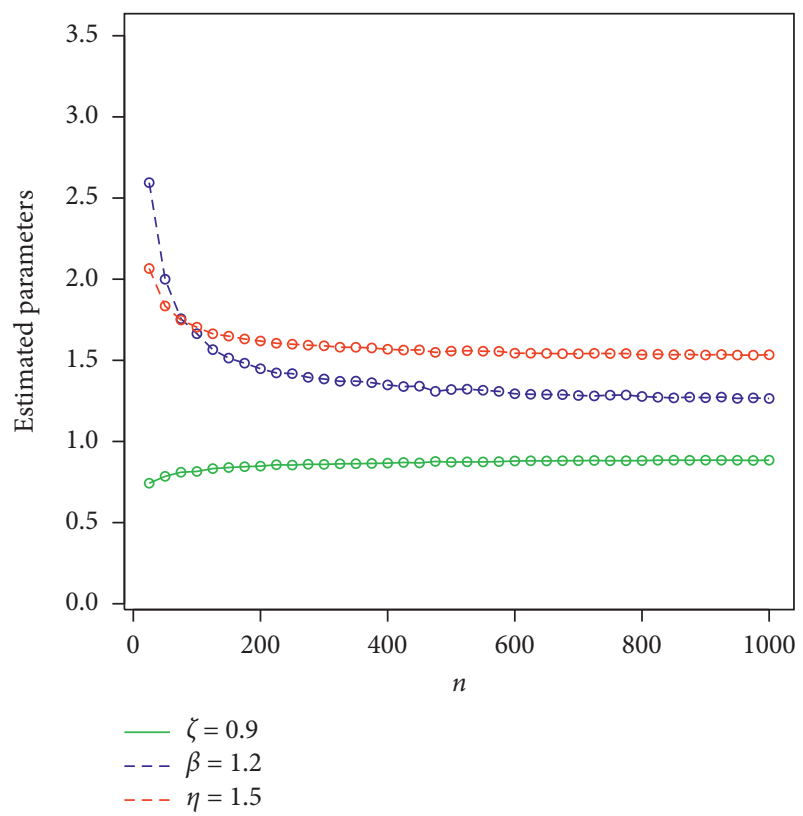

(a)

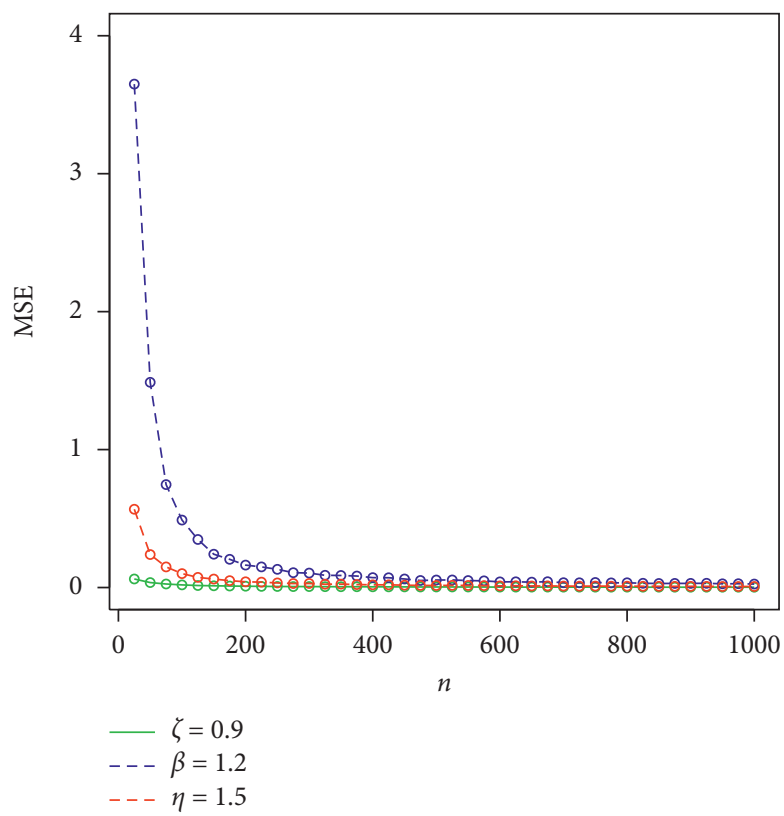

(b)

Figure 6: Graphical display of the simulation results provided in Table 1. (a) Plot of estimated parameters vs. $n$. (b) Plot of MSEs vs. $n$.

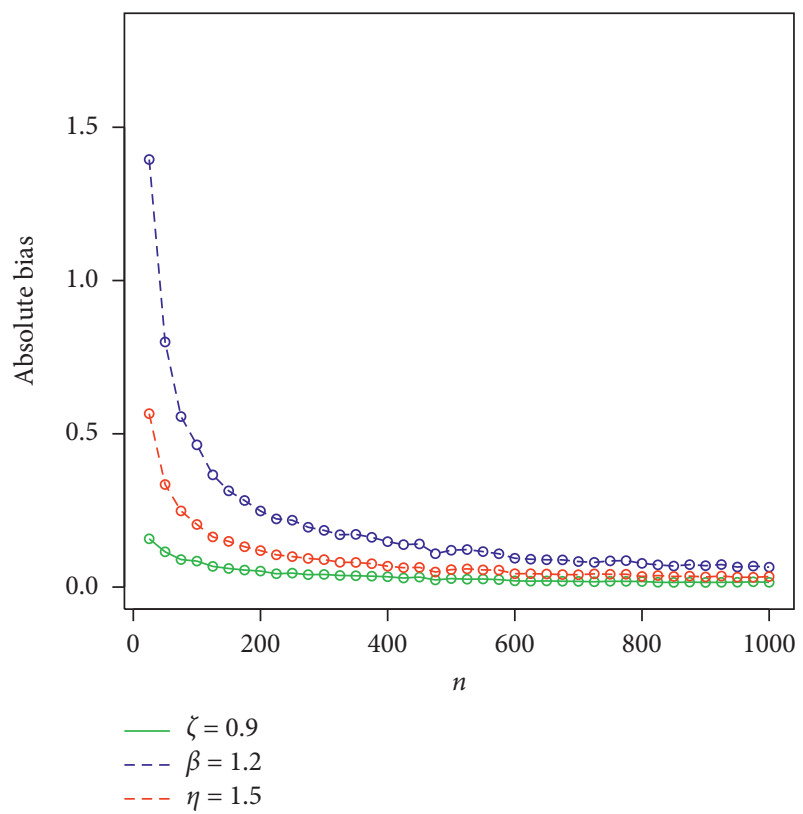

(a)

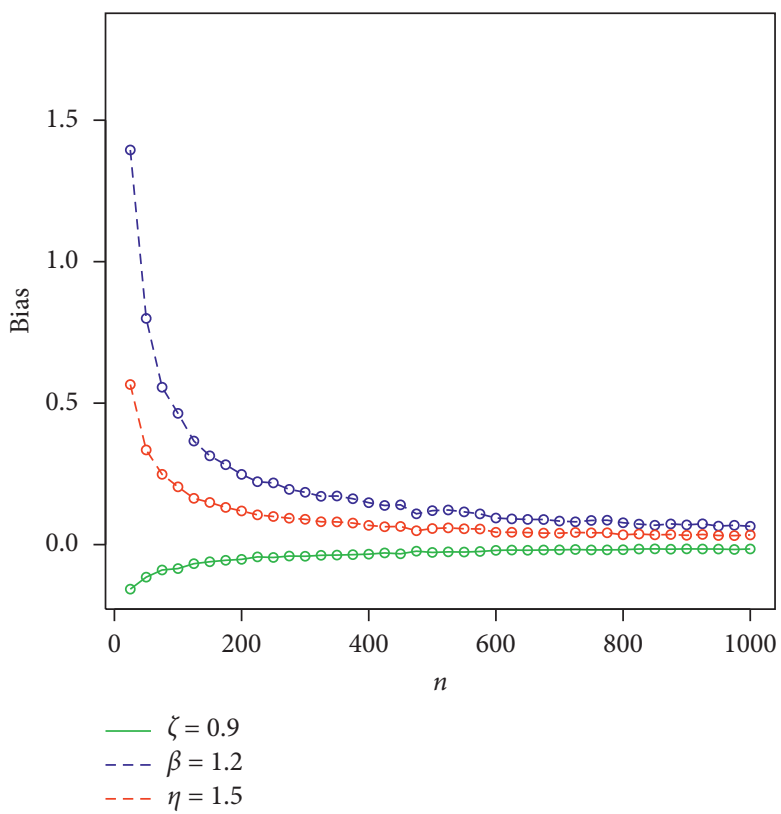

(b)

Figure 7: Graphical display of the simulation results provided in Table 1. (a) Plot of absolute blases vs. $n$. (b) Plot of blases vs. $n$.

$$
\operatorname{TVP}_{q}(X)=\mathrm{TVaR}_{q}+\delta \mathrm{TV}_{q},
$$

where $0<\delta<1$. By substituting (30) and (31) in (34), we can get the TVP of the proposed distribution.

7.5. Numerical Study of the Risk Measures. The importance of this subsection is due to the numerical study of $\mathrm{VaR}$ and TVaR for the Weibull and HTBPT-Weibull distributions for different sets of parameters. The followed steps are given by
(1) A random sample of size $n=100$ is generated from the Weibull and HTBPT-Weibull distributions, and the maximum likelihood method is adopted to estimate the model parameters.

(2) Repetitions of 1000 iterations are made to find the values of $\mathrm{VaR}$ and $\mathrm{TVaR}$ for these distributions

Tables 3-5 contain the simulation results of the risk measures. The simulation results are also displayed graphically corresponding to each table in Figures 10-12, respectively. 


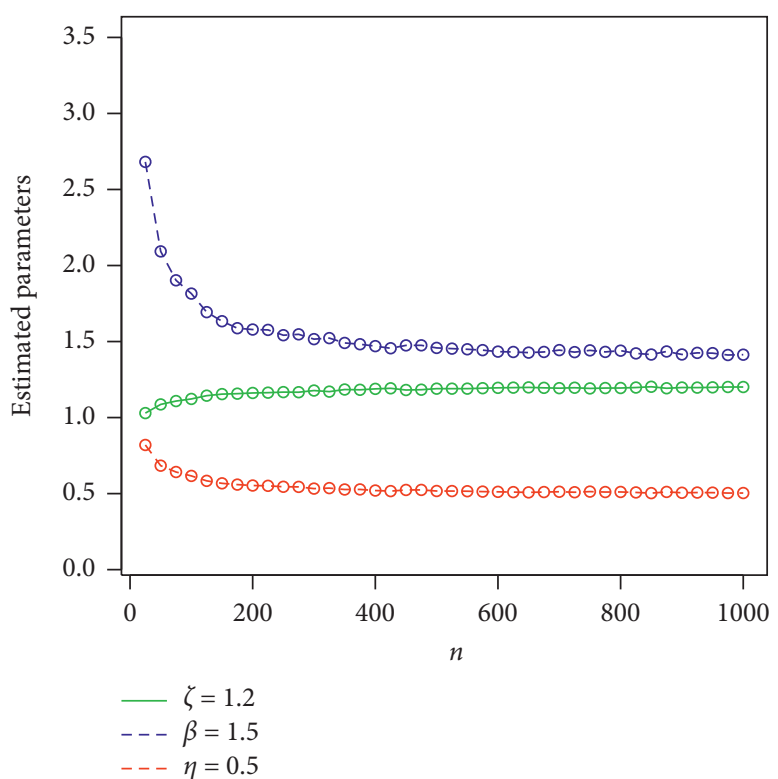

(a)

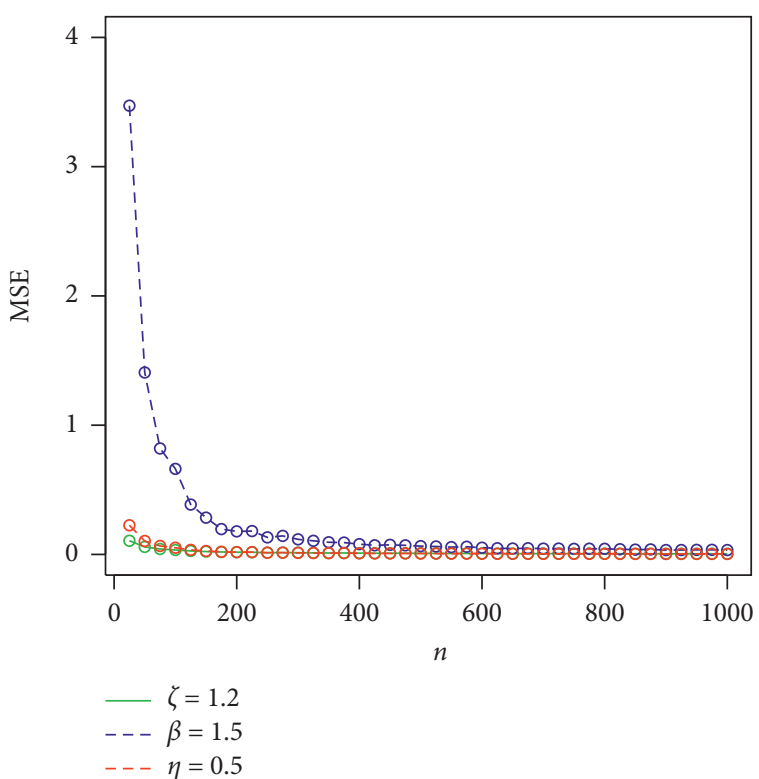

(b)

FIgURE 8: Graphical display of the simulation results provided in Table 2. (a) Plot of estimated parameters vs. $n$. (b) Plot of MSEs vs. $n$.

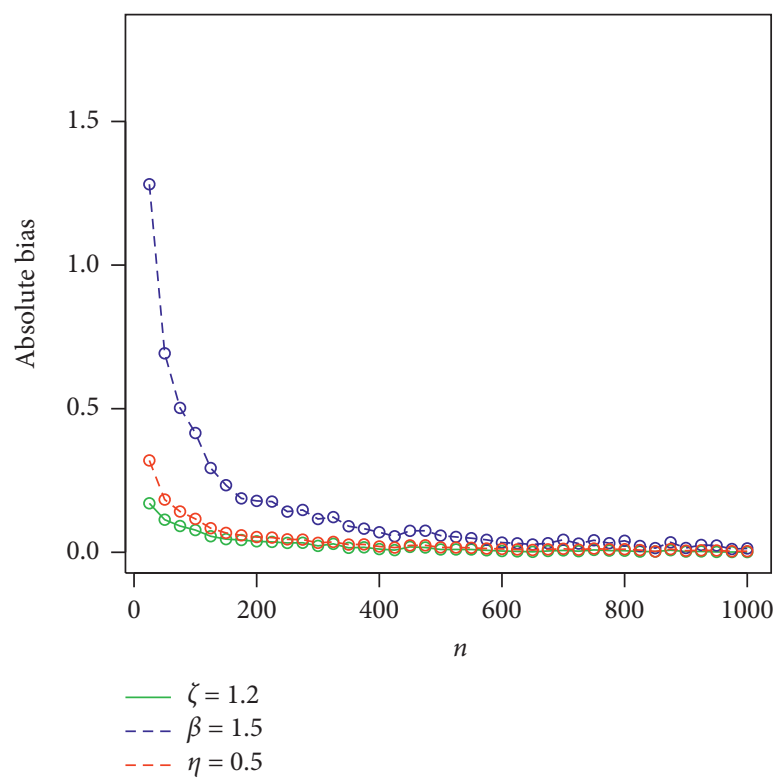

(a)

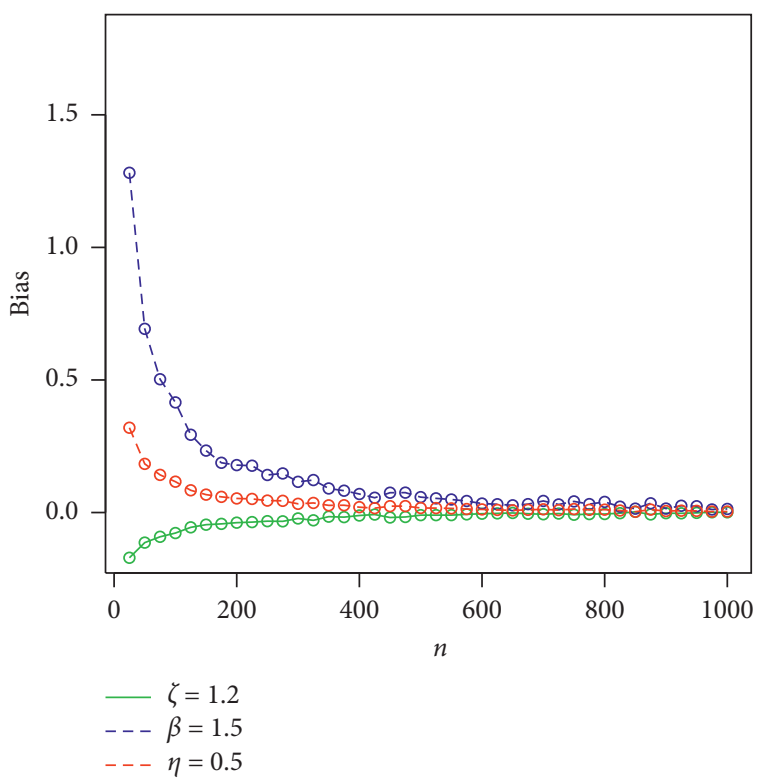

(b)

FIgUre 9: Graphical display of the simulation results provided in Table 2. (a) Plot of absolute biases vs. $n$. (b) Plot of biases vs. $n$.

After performing the simulation study based on VaR and TVaR, we observed that the proposed model is better than the other compared models because it has higher values of the risk measures (VaR and TVaR).

\section{Practical Illustration of the Proposed Model}

This section deals with the practical illustration of the proposed model using a real-life insurance dataset.
Additionally, we use the same data to calculate the actuarial measures of the Weibull and HTBPT-Weibull distributions.

8.1. An Application to Vehicle Insurance Loss Data. In this part of the study, we consider a dataset from insurance sciences to show the practicability of the proposed distribution. These data have also been studied by Zhao et al. [23] and can be found at http://www.businessandeconomics.mq. edu.au. The comparison of the HTBPT-Weibull distribution 
TABLE 3: For $n=100$, the simulation study of the risk measures for Weibull and HTBPT-Weibull distributions.

\begin{tabular}{|c|c|c|c|c|}
\hline Distributions & Parameters & Significance level & VaR & TVaR \\
\hline \multirow{8}{*}{ Weibull } & \multirow{4}{*}{$\alpha=0.8$} & 0.700 & 1.6688 & 2.2356 \\
\hline & & 0.750 & 1.7953 & 2.3365 \\
\hline & & 0.800 & 1.9397 & 2.4542 \\
\hline & & 0.850 & 2.1122 & 2.5980 \\
\hline & \multirow{4}{*}{$\gamma=1$} & 0.900 & 2.3352 & 2.7880 \\
\hline & & 0.950 & 2.6763 & 3.0863 \\
\hline & & 0.975 & 2.9810 & 3.3589 \\
\hline & & 0.999 & 4.1260 & 4.4174 \\
\hline \multirow{8}{*}{ HTBPT-Weibull } & \multirow{3}{*}{$\alpha=0.8$} & 0.700 & 2.3073 & 5.0387 \\
\hline & & 0.750 & 2.6034 & 5.5565 \\
\hline & & 0.800 & 2.9903 & 6.2489 \\
\hline & \multirow[t]{2}{*}{$\beta=2$} & 0.850 & 3.5376 & 7.2499 \\
\hline & & 0.900 & 4.4248 & 8.9054 \\
\hline & \multirow{3}{*}{$\gamma=1$} & 0.950 & 6.3569 & 12.5802 \\
\hline & & 0.975 & 9.0147 & 17.6978 \\
\hline & & 0.999 & 43.6677 & 85.0932 \\
\hline
\end{tabular}

TABLE 4: For $n=100$, the simulation study of the risk measures for Weibull and HTBPT-Weibull distributions.

\begin{tabular}{lcccc}
\hline Distributions & Parameters & $\begin{array}{c}\text { Significance } \\
\text { level }\end{array}$ & VaR & TVaR \\
\hline \multirow{4}{*}{ Weibull } & & 0.700 & 2.5628 & 4.9448 \\
& & 0.750 & 2.9824 & 5.3806 \\
& & 0.800 & 3.5015 & 5.9178 \\
& & 0.850 & 4.1788 & 6.6160 \\
& & 0.900 & 5.1464 & 7.6095 \\
& & 0.950 & 6.8296 & 9.3302 \\
& & 0.975 & 8.5426 & 11.0747 \\
& & 0.999 & 16.7703 & 19.4062 \\
\hline \multirow{3}{*}{$\alpha=0.5$} & 0.700 & 4.1839 & 9.4191 \\
& & 0.750 & 4.7366 & 10.4131 \\
& & 0.800 & 5.4611 & 11.7463 \\
& & 0.850 & 6.4904 & 13.6812 \\
& & 0.900 & 8.1681 & 16.8977 \\
& $\gamma=1$ & 0.950 & 11.8517 & 24.0937 \\
& & 0.975 & 16.9687 & 34.2111 \\
& & 0.999 & 85.8277 & 171.7096 \\
\hline
\end{tabular}

is made with the other well-known distributions including the two-parameter Weibull, Lomax, Burr-XII (B-XII), threeparameter new beta-power transformed Weibull (NBPTW), four-parameter Kumaraswamy-Weibull $(\mathrm{Ku}-\mathrm{W})$, and new Weibull Burr-XII (NWB-XI) distributions. The cdfs of the competing distributions are

(1) Weibull distribution

$$
G(x ; \alpha, \gamma)=1-e^{-\gamma x^{\alpha}}, \quad x>0, \alpha, \gamma>0 .
$$

(2) W-Loss distribution

$$
G(x ; \alpha, \gamma, \sigma)=1-\frac{\sigma e^{-\gamma x^{\alpha}}}{\sigma+\gamma x^{\alpha}}, \quad x>0, \alpha, \gamma, \sigma>0 .
$$

(3) NBPTW distribution
TABLE 5: For $n=100$, the simulation study of the risk measures for

\begin{tabular}{|c|c|c|c|c|}
\hline Distributions & Parameters & $\begin{array}{c}\text { Significance } \\
\text { level }\end{array}$ & VaR & TVaR \\
\hline \multirow{8}{*}{ Weibull } & \multirow{4}{*}{$\alpha=1.4$} & 0.700 & 3.5223 & 6.5398 \\
\hline & & 0.750 & 4.0674 & 7.0905 \\
\hline & & 0.800 & 4.7365 & 7.7658 \\
\hline & & 0.850 & 5.6019 & 8.6383 \\
\hline & \multirow{4}{*}{$\gamma=1$} & 0.900 & 6.8261 & 9.8712 \\
\hline & & 0.950 & 8.9288 & 11.9865 \\
\hline & & 0.975 & 11.0415 & 14.1096 \\
\hline & & 0.999 & 20.9426 & 24.0443 \\
\hline \multirow{8}{*}{ HTBPT-Weibull } & \multirow{3}{*}{$\alpha=1.4$} & 0.700 & 5.2740 & 11.8733 \\
\hline & & 0.750 & 5.9707 & 13.1263 \\
\hline & & 0.800 & 6.8839 & 14.8067 \\
\hline & \multirow[t]{2}{*}{$\beta=0.9$} & 0.850 & 8.1815 & 17.2459 \\
\hline & & 0.900 & 10.2962 & 21.3004 \\
\hline & \multirow{3}{*}{$\gamma=1$} & 0.950 & 14.9397 & 30.3714 \\
\hline & & 0.975 & 21.3899 & 43.1248 \\
\hline & & 0.999 & 108.1901 & 216.4486 \\
\hline
\end{tabular}
Weibull and HTBPT-Weibull distributions.

$$
G(x ; \alpha, \beta, \gamma)=\frac{\beta^{\left(1-e^{-\gamma x^{\alpha}}\right)}-e^{-\gamma x^{\alpha}}}{\beta}, \quad x>0, \alpha, \beta, \gamma>0, \beta \neq 1 .
$$

(4) Kumaraswamy-Weibull

$$
G(x ; \alpha, \gamma, c, t)=1-\left(1-\left(1-e^{-\gamma x^{\alpha}}\right)^{c}\right)^{t}, \quad x>0, \alpha, \gamma, c, t>0 .
$$

(5) Lomax

$$
G(x ; \alpha, \gamma)=1-\left(1+\frac{x}{\gamma}\right)^{-\alpha}, \quad x>0, \alpha, \gamma>0 .
$$

(6) NWB-XII 


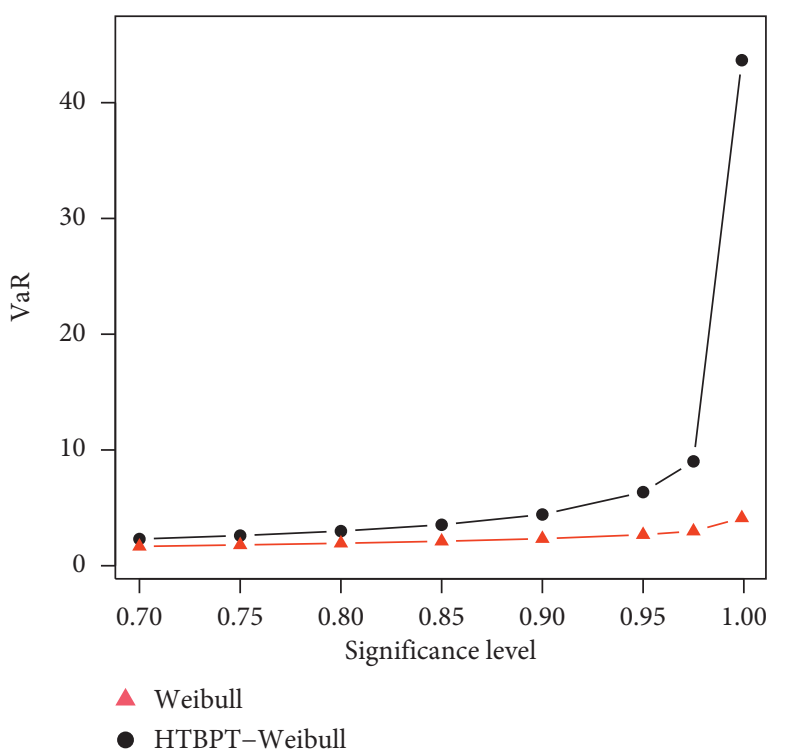

(a)

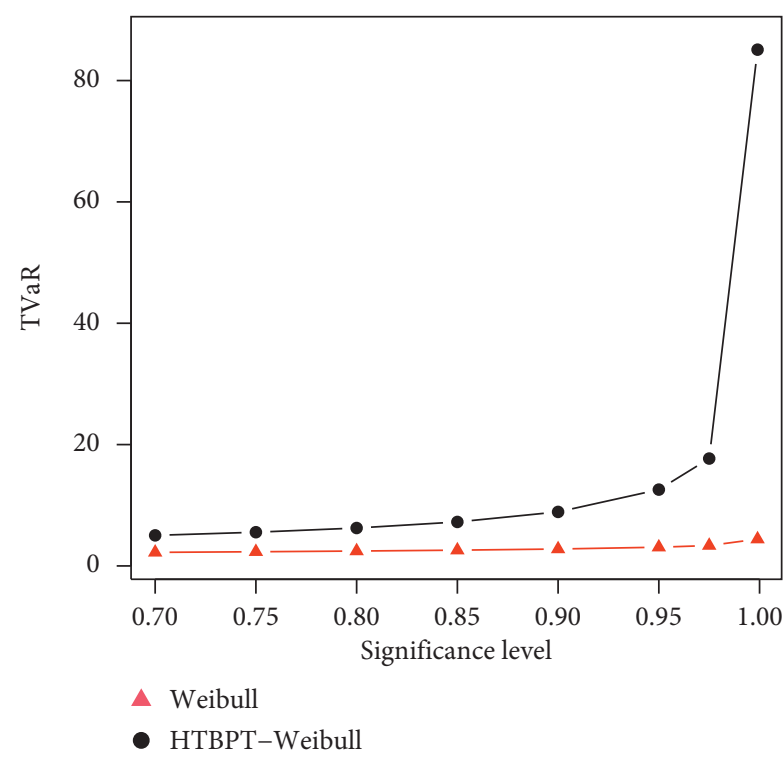

(b)

Figure 10: Graphical sketching of the numerical results provided in Table 3.

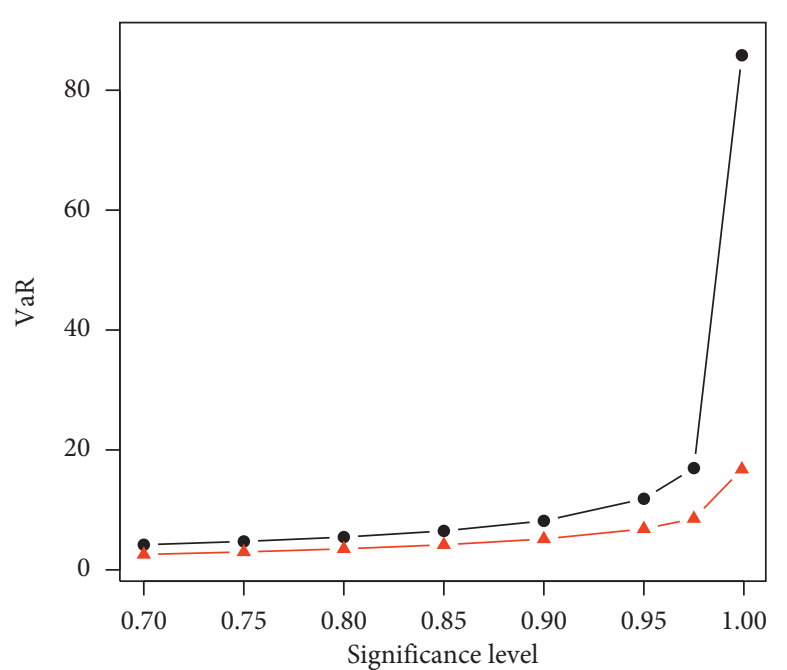

- Weibull

- HTBPT-Weibull

(a)

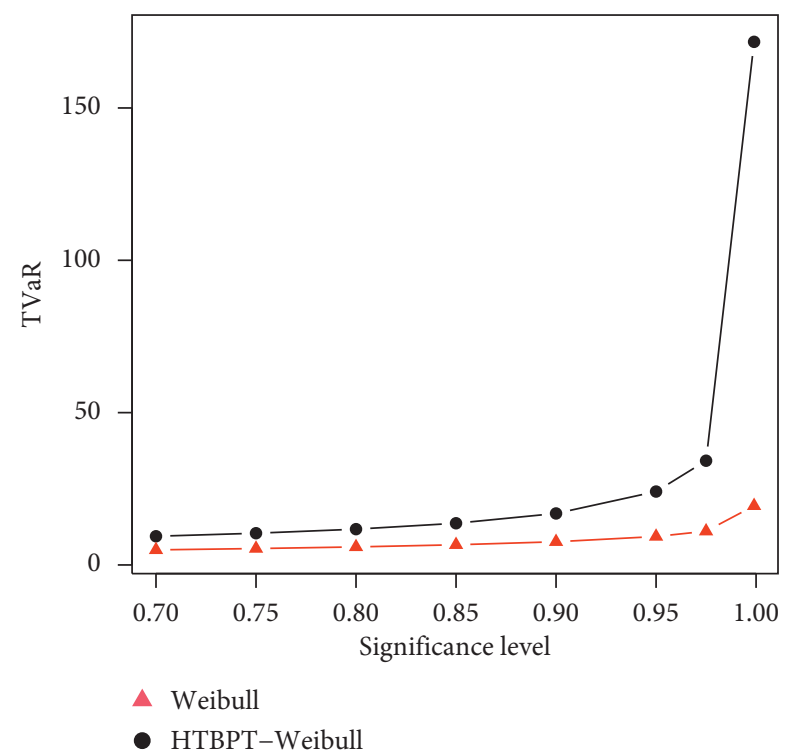

(b)

Figure 11: Graphical sketching of the numerical results provided in Table 4.

$G(x ; \alpha, \gamma, c, t)=1-\exp \left(-\alpha\left(t \log \left(1+x^{c}\right)\right)^{\gamma}\right), \quad x>0, c, t, \alpha, \gamma>0$.

(7) B-XII

$$
G(x ; c, t)=1-\left(1+x^{c}\right)^{-t}, \quad x>0, c, t>0 .
$$

The comparison is made using some certain analytical measures called the Akaike information criterion (AIC) (Akaike [24]), Bayesian information criterion (BIC) (Schwarz [25]), Hannan-Quinn information criterion
(HQIC) (Hannan and Quinn [26]), and consistent Akaike information criterion (CAIC) (Bozdogan [27]). These measures are as follows:

The AIC is given by

$$
\mathrm{AIC}=2 k-2 \ell .
$$

The CAIC is

$$
\mathrm{CAIC}=\frac{2 n k}{n-k-1}-2 \ell .
$$




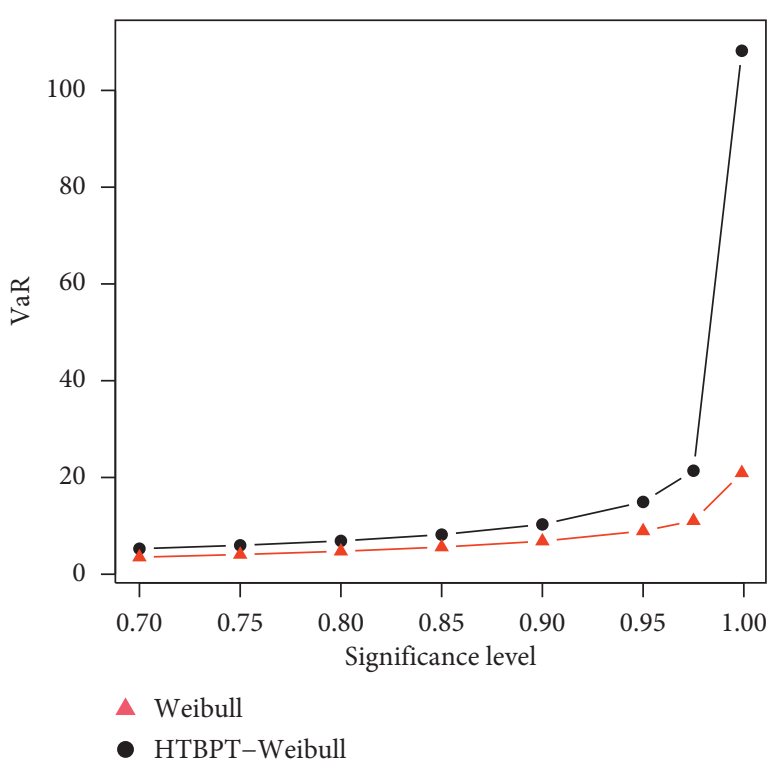

(a)

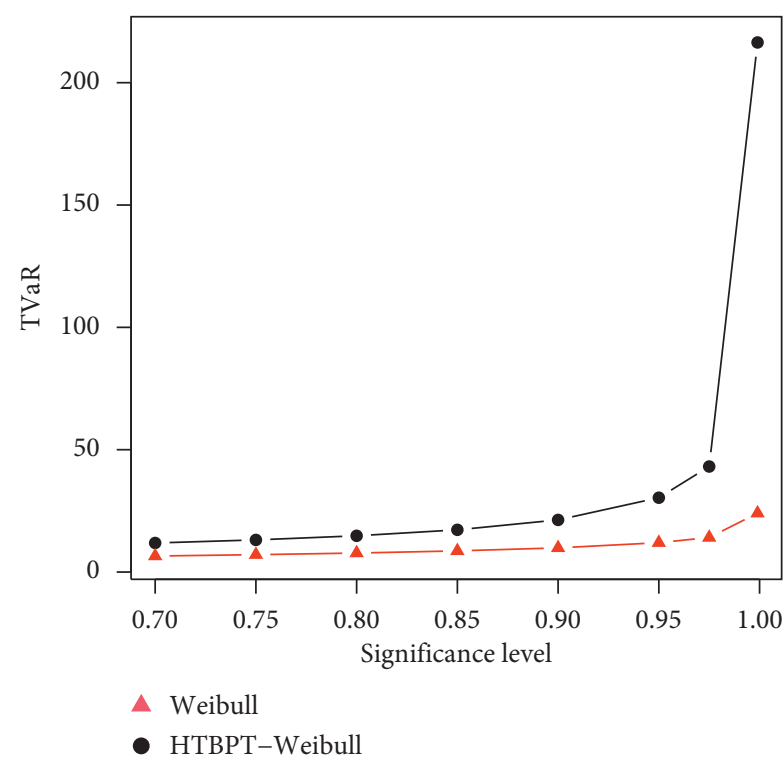

(b)

Figure 12: Graphical sketching of the numerical results provided in Table 5.

TABLE 6: The MLEs of the competing models for the vehicle insurance loss data.

\begin{tabular}{lccccc}
\hline Distributions & $\widehat{\alpha}$ & $\widehat{\beta}$ & $\widehat{\gamma}$ & $\widehat{c}$ & $\widehat{t}$ \\
\hline HTBPT-Weibull & 0.99 & 1.06 & 0.004 & & \\
Weibull & 1.01 & & 0.003 & & \\
W-Loss & 0.94 & & 1.29 & & \\
Ku-W & 0.87 & 1.18 & 0.008 & 1.50 & \\
NBPTW & 0.90 & & 0.86 & \\
Lomax & 0.49 & & 30.00 & \\
NWB-XII & 0.08 & & 25.55 & 0.06 & 1.22 \\
B-XII & & & 0.04 & 4.42 \\
\hline
\end{tabular}

The BIC is calculated as follows:

$$
\mathrm{BIC}=k \log (n)-2 \ell .
$$

The HQIC is

$$
\mathrm{HQIC}=2 k \log (\log (n))-2 \ell,
$$

where $k$ is the number of model parameters, $n$ is the sample size, and $\ell$ refers to the log-likelihood function evaluated at MLEs.

For the calculation of the analytical measures, we use the optim $\mathrm{R}$ function with the argument method= "SANN." Smaller values of these analytical measures indicate the best fit. The MLEs of the model parameters along with the analytical measures are reported in Tables 6 and 7, respectively. Referring to the results provided in Table 7, we found that the HTBPT-Weibull distribution is a superior model and provides the best fit among the other considered competitors. In support of Table 7, the estimated pdf and cdf of the proposed distribution are shown in Figure 13, which closely fit the proposed model. The probability-probability (PP) plot and Kaplan-Meier survival plot of the HTBPT-Weibull model are shown in Figure 14. From the PP and Kaplan-Meier survival plots, it is clear that the proposed is a good candidate model for modeling heavy-tailed insurance datasets. Furthermore, the quantile-quantile (QQ) plot and box plot of the data are shown in Figure 15.

8.2. Computation of the Actuarial Measures Using Real Data. In this subsection, we calculate and compare the values of the VaR and TVaR measures of the Weibull and HTBPT-Weibull distributions using the data discussed in subsection 8.1. The numerical results are reported in Table 8.

\section{Concluded Remarks}

The statistical distributions are very useful for modeling financial and actuarial datasets. Among them, the heavytailed distributions are very prominent for modeling financial and other related datasets. A number of methods to define heavy-tailed distributions have been studied in the literature. However, unfortunately, these methods still have 
Table 7: Analytical measures of the competing models for the vehicle insurance loss data.

\begin{tabular}{lcccc}
\hline Distributions & AIC & BIC & CIAC & HQIC \\
\hline HTBPT-Weibull & 430.6 & 435.0 & 431.4 & 432.0 \\
Weibull & 432.3 & 439.2 & 434.0 & 435.7 \\
W-Loss & 432.1 & 437.5 & 433.7 & 434.9 \\
Ku-W & 432.2 & 438.1 & 433.7 & 434.1 \\
NBPTW & 434.8 & 440.0 & 435.5 & 437.2 \\
Lomax & 460.1 & 463.0 & 460.5 & 461.1 \\
NWB-XII & 431.7 & 437.6 & 433.2 & 433.6 \\
B-XII & 503.4 & 506.3 & 503.8 & 504.4 \\
\hline
\end{tabular}

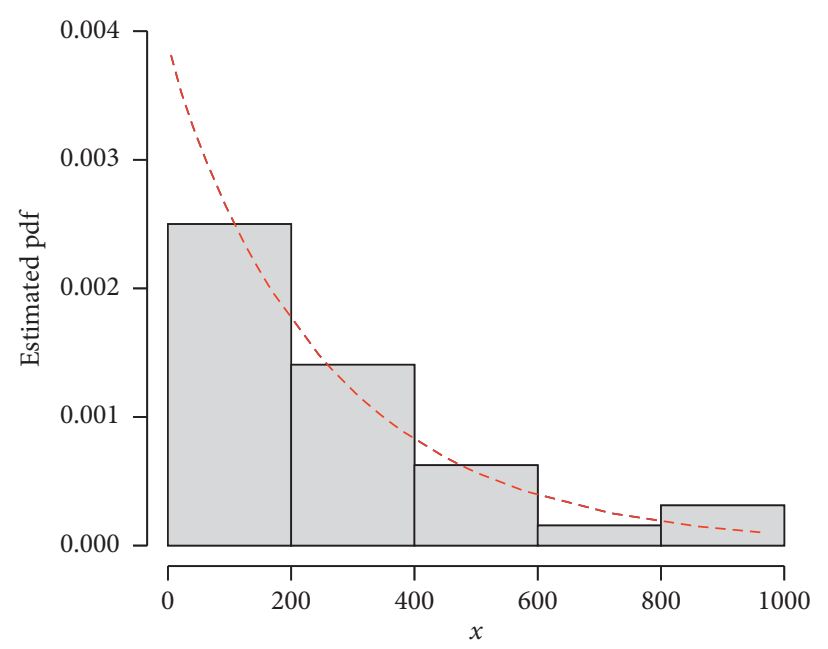

(a)

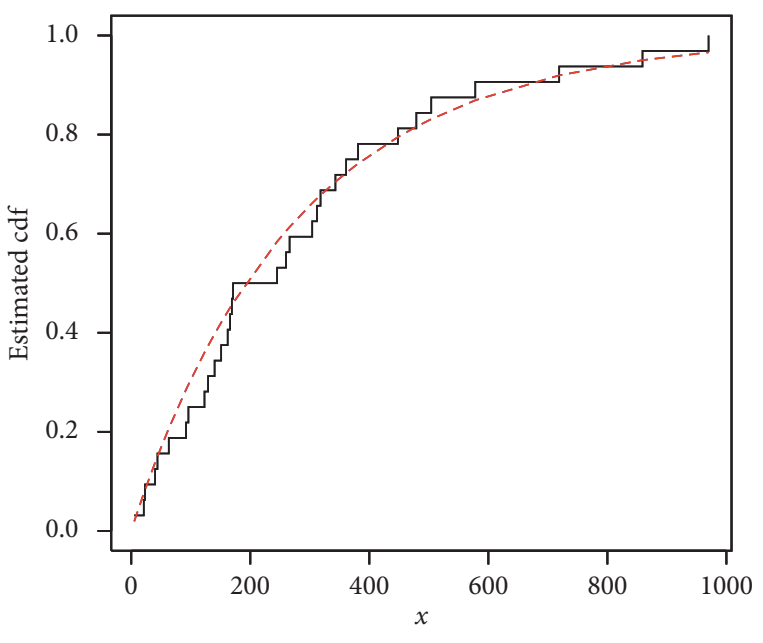

(b)

FIgURE 13: The estimated pdf and cdf of the HTBPT-Weibull distribution for the vehicle insurance loss data.

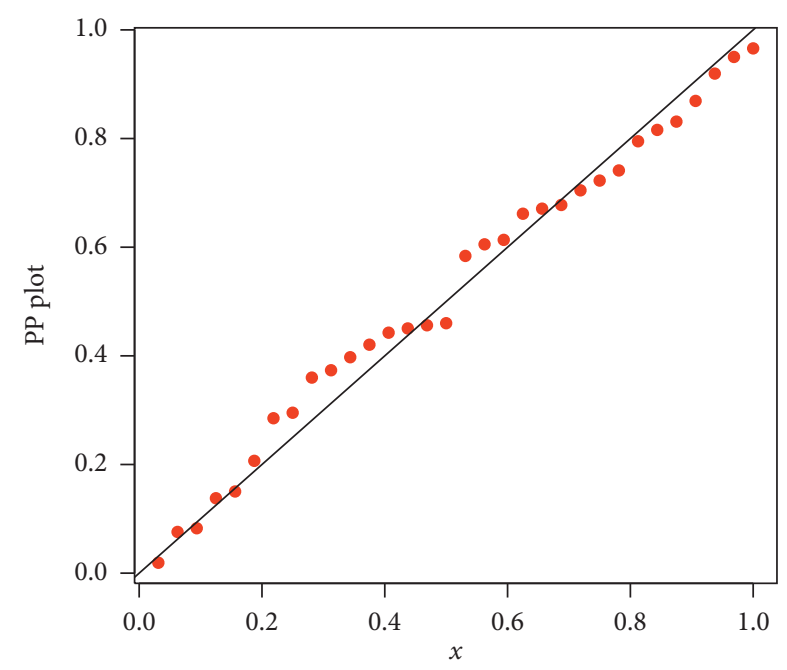

(a)

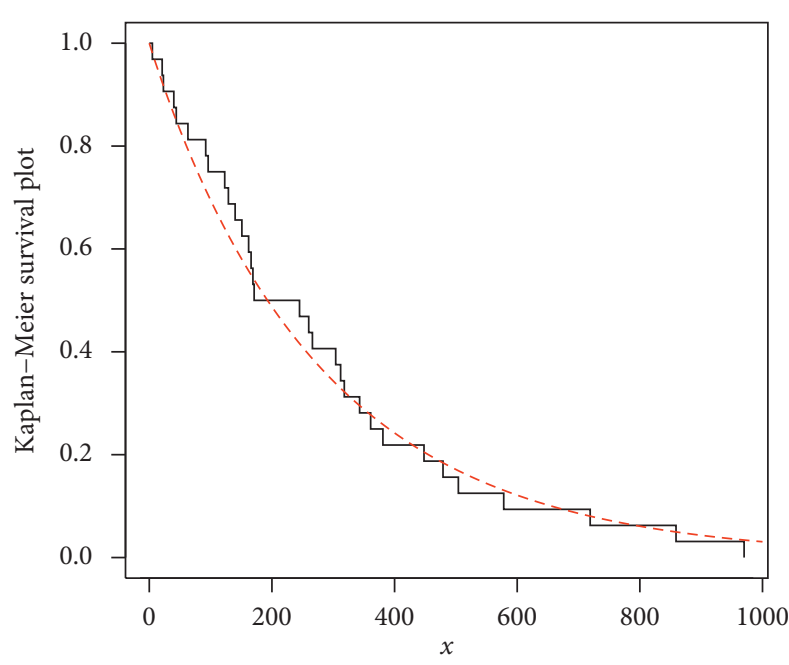

(b)

Figure 14: The PP and Kaplan-Meier survival survival plots of the HTBPT-Weibull distribution for the vehicle insurance loss data.

certain limitations. To address the issues associated with the former methods, we introduced a new heavy-tailed family of distributions. By using the proposed method, a new threeparameter special model, called the heavy-tailed beta power transformed Weibull distribution is studied in detail. Actuarial measures of the proposed model are calculated, and a simulation study is conducted to show the usefulness of the proposed method in actuarial sciences. The applicability of 


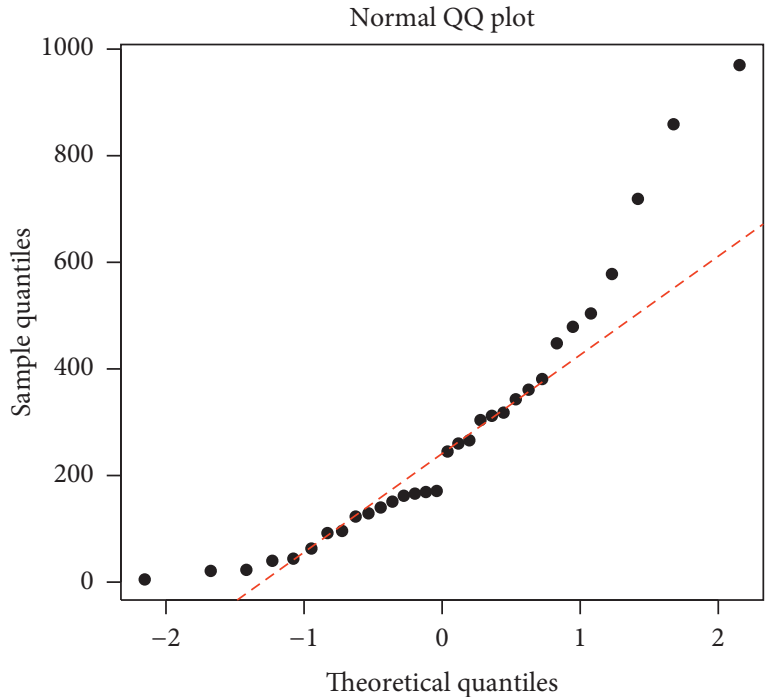

(a)

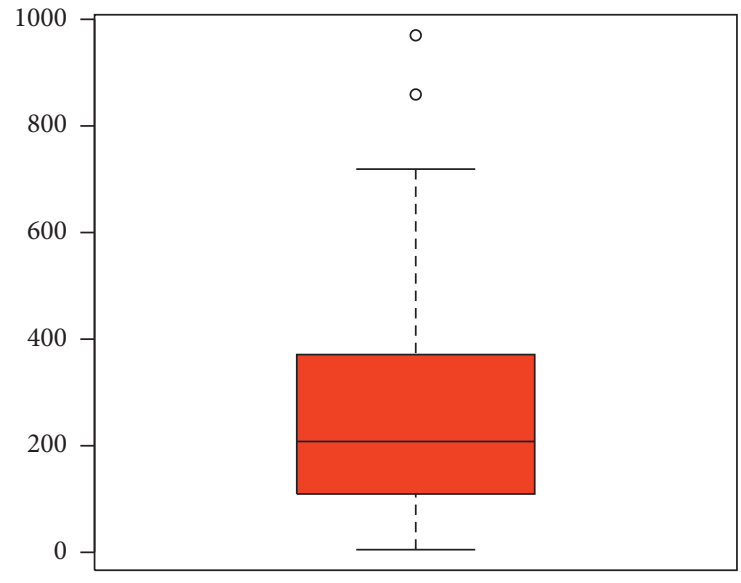

(b)

FIgURE 15: The QQ and box plots of the vehicle insurance loss data.

TABLE 8: Results for the actuarial measures using the vehicle insurance loss data.

\begin{tabular}{|c|c|c|c|c|}
\hline Distributions & Parameters & $\begin{array}{c}\text { Significance } \\
\text { level }\end{array}$ & VaR & TVaR \\
\hline \multirow{8}{*}{ Weibull } & \multirow{4}{*}{$\widehat{\alpha}=1.012$} & 0.700 & 326.949 & 593.777 \\
\hline & & 0.750 & 375.850 & 642.400 \\
\hline & & 0.800 & 435.601 & 701.844 \\
\hline & & 0.850 & 512.495 & 778.385 \\
\hline & \multirow{4}{*}{$\widehat{\gamma}=0.003$} & 0.900 & 620.646 & 886.105 \\
\hline & & 0.950 & 805.042 & 1069.886 \\
\hline & & 0.975 & 988.944 & 1253.282 \\
\hline & & 0.999 & 1838.590 & 2101.309 \\
\hline \multirow{8}{*}{ HTBPT-Weibull } & \multirow{3}{*}{$\widehat{\alpha}=0.991$} & 0.700 & 335.147 & 691.138 \\
\hline & & 0.750 & 386.355 & 748.644 \\
\hline & & 0.800 & 449.103 & 819.083 \\
\hline & \multirow{2}{*}{$\widehat{\beta}=1.061$} & 0.850 & 530.105 & 909.978 \\
\hline & & 0.900 & 644.443 & 1038.238 \\
\hline & \multirow{3}{*}{$\widehat{\gamma}=0.004$} & 0.950 & 840.290 & 1257.859 \\
\hline & & 0.975 & 1036.531 & 1477.869 \\
\hline & & 0.999 & 1951.423 & 2503.161 \\
\hline
\end{tabular}

the family of distributions has been illustrated using a reallife heavy-tailed dataset from insurance and shown that the proposed model performs reasonably well compared to other well-known heavy-tailed distributions.

\section{Appendix}

\section{$\boldsymbol{R}$ Code for Analysis}

Note: in the following R-code, pm is used for the proposed model.

data $<$-read.csv(file.choose(), header=TRUE)

data $=$ data[,1]

data $=$ data[!is.na(data) $]$

data
Iparaindent $\quad$ \#\#\#\#\#\#\#\#\#\#\#\#\#\#\#\#\#\#\#\#\#\# \#\#\#\#\#\#\#\#\#\#\#\#\#\#\#\#\#\#\#\#\#\#

\#\#\#\#\#\#\#\#\#\#\#\#\#\# PDF of the proposed model

\#\#\#\#\#\#\#\#\#\#\#\#\#\#\#\#\#\#\#\#\#\#\#\#\#\#\#\#\#\#\#\#\#\#\#\# \#\#\#\#\#\#\#\#

pdf_pm $<-$ function(par,x)

\{

alpha=par [1]

gamma=par [2]

beta=par [3]

alpha $*$ gamma $*(\hat{x}($ alpha -1$)) * \exp (-$

gamma $* \hat{x a l p h a)}$

$<\mathrm{v}$ :shape xmlns:wpc="http://schemas.microsoft.com/office/word/2010/wordpro-

cessingCanvas"xmlns:mc="http://schemas.

openxmlformats.org/markup-compatibility/2006” xmlns:w14="http://schemas.microsoft.com/office/word/2010/wordml" xmlns:wpg="http://schemas.microsoft. com/office/word/2010/wordprocessingGroup" xmlns: wpi="http://schemas.microsoft.com/office/word/2010/ wordprocessingInk" xmlns:wps="http://schemas.microsoft.com/office/word/2010/wordprocessingShape" id =“_x0000_i1910" type=“\#_x0000_t75" style="width: 6.8pt;height:14.75pt" o:ole $=$ "” $><\mathrm{v}$ :imagedata $\mathrm{r}: \mathrm{id}=$ "rId554" o:title="”/ $><$ o:OLEObject xmlns:wpc= "http://schemas.microsoft.com/office/word/2010/wordprocessingCanvas" xmlns:mc="http://schemas.open xmlformats.org/markup-compatibility/2006” xmlns: w14="http://schemas.microsoft.com/office/word/2010 /wordml" xmlns:wpg="http://schemas.microsoft.com/ office/word/2010/wordprocessingGroup" xmlns:wpi= "http://schemas.microsoft.com/office/word/2010/word processingInk" xmlns:wps="http://schemas.microsoft. com/office/word/2010/wordprocessingShape" Type= 


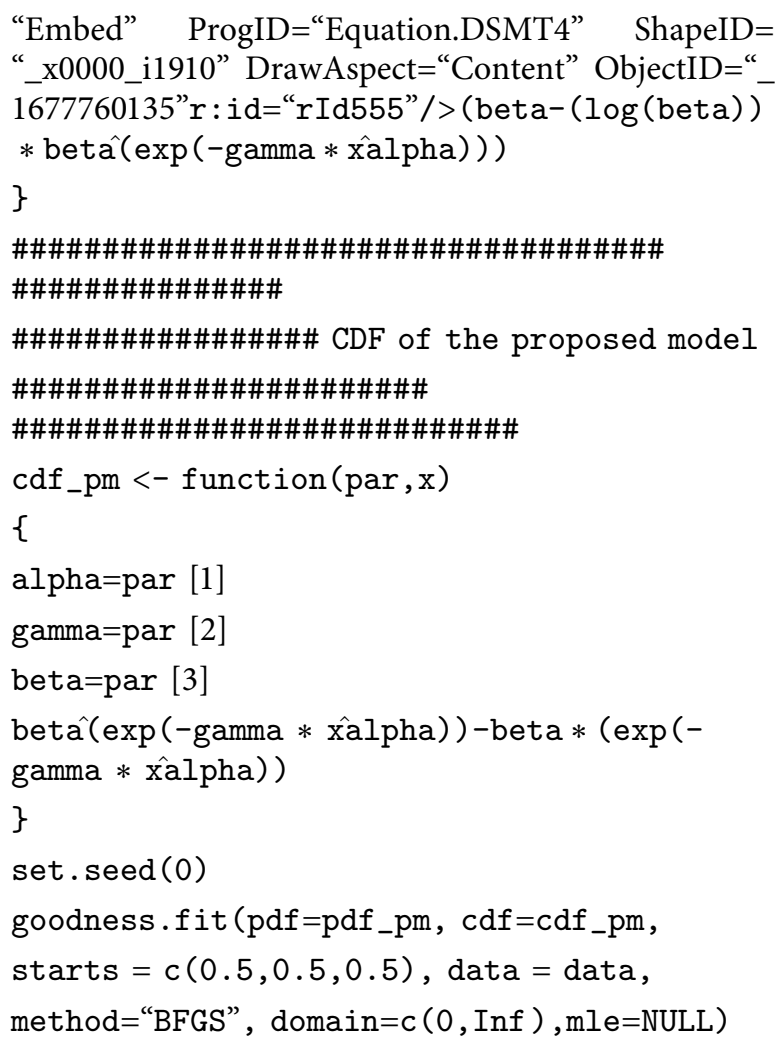

\section{Data Availability}

The link of the dataset used in this study is included within the article, and the data are available from Zubair Ahmad upon request.

\section{Conflicts of Interest}

The authors declare that they have no conflicts of interest.

\section{References}

[1] R. Kazemi and M. Noorizadeh, "A comparison between skewlogistic and skew-normal distributions," Matematika, vol. 31, pp. 15-24, 2015.

[2] C. Adcock, M. Eling, and N. Loperfido, "Skewed distributions in finance and actuarial science: a review," The European Journal of Finance, vol. 21, pp. 1253-1281, 2015.

[3] D. Bhati and S. Ravi, "On generalized log-Moyal distribution: a new heavy tailed size distribution," Insurance: Mathematics and Economics, vol. 79, pp. 247-259, 2018.

[4] Z. Ahmad, G. G. Hamedani, and N. S. Butt, "Recent developments in distribution theory: a brief survey and some new generalized classes of distributions," Pakistan Journal of Statistics and Operation Research, vol. 15, pp. 87-110, 2019.

[5] Z. Ahmad, E. Mahmoudi, G. G. Hamedani, and G. Hamedani, "A class of claim distributions: properties, characterizations and applications to insurance claim data," Communications in Statistics - Theory and Methods, vol. 49, pp. 1-26, 2020.

[6] Z. Ahmad, E. Mahmoudi, and M. Alizadeh, "Modelling insurance losses using a new beta power transformed family of distributions," Communications in Statistics - Simulation and Computation, vol. 49, pp. 1-22, 2020.

[7] Z. Ahmad, E. Mahmoudi, and O. Kharazmi, "On modeling the earthquake insurance data via a new member of the T-X family," Computational Intelligence and Neuroscience, vol. 2020, Article ID 7631495, 20 pages, 2020.

[8] K. Cooray and M. Ananda, "Modeling actuarial data with a composite lognormal-Pareto model," Scandinavian Actuarial Journal, vol. 5, pp. 321-334, 2005.

[9] D. P. M. Scollnik, "On composite lognormal-Pareto models," Scandinavian Actuarial Journal, vol. 2007, no. 1, pp. 20-33, 2007.

[10] R. V. Hogg and S. A. Klugman, Loss Distributions, p. 249, Wiley, New York, NY, USA, 2009.

[11] S. A. Klugman, H. H. Panjer, and G. E. Willmot, Loss Models: From Data to Decisions, John Wiley \& Sons, Hoboken, NJ, USA, 2012.

[12] S. Nadarajah and S. A. A. Bakar, "New composite models for the Danish fire insurance data," Scandinavian Actuarial Journal, vol. 2014, no. 2, pp. 180-187, 2014.

[13] S. A. Bakar, N. A. Hamzah, M. Maghsoudi, and S. Nadarajah, "Modelling loss data using composite models," Insurance: Mathematics and Economics, vol. 61, pp. 146-154, 2015.

[14] T. Shushi, "Skew-elliptical distributions with applications in risk theory," European Actuarial Journal, vol. 7, no. 1, pp. 277-296, 2017.

[15] A. Punzo, "A new look at the inverse Gaussian distribution with applications to insurance and economic data," Journal of Applied Statistics, vol. 46, no. 7, pp. 1260-1287, 2019.

[16] A. Punzo, A. Mazza, and A. Maruotti, "Fitting insurance and economic data with outliers: a flexible approach based on finite mixtures of contaminated gamma distributions," Journal of Applied Statistics, vol. 45, no. 14, pp. 2563-2584, 2018.

[17] A. Punzo, L. Bagnato, and A. Maruotti, "Compound unimodal distributions for insurance losses," Insurance: Mathematics and Economics, vol. 81, pp. 95-107, 2018.

[18] D. Bhati and S. Ravi, "On generalized log-Moyal distribution: a new heavy tailed size distribution," Mathematics and Economics, vol. 79, pp. 247-259, 2015.

[19] Z. Ahmad, E. Mahmoudi, S. Dey, and S. K. Khosa, "Modeling vehicle insurance loss data using a new member of T-X family of distributions," Journal of Statistical Theory and Applications, vol. 19, no. 2, pp. 133-147, 2020.

[20] Z. Ahmad, E. Mahmoudi, G. G. Hamedani, and O. Kharazmi, "New methods to define heavy-tailed distributions with applications to insurance data," Journal of Taibah University for Science, vol. 14, no. 1, pp. 359-382, 2020.

[21] A. Alzaatreh, C. Lee, and F. Famoye, "A new method for generating families of continuous distributions," Metron, vol. 71, no. 1, pp. 63-79, 2013.

[22] P. Artzner, "Application of coherent risk measures to capital requirements in insurance," North American Actuarial Journal, vol. 3, pp. 1-25, 1999.

[23] W. Zhao, S. K. Khosa, Z. Ahmad, M. Aslam, and A. Z. Afify, "Type-I heavy tailed family with applications in medicine, engineering and insurance," Plos One, vol. 15, Article ID e0237462, 2020.

[24] H. Akaike, "A new look at the statistical model identification," IEEE Transactions on Automatic Control, vol. 19, no. 6, pp. 716-723, 1974.

[25] G. Schwarz, "Estimating the dimension of a model," The Annals of Statistics, vol. 6, pp. 461-464, 1978. 
[26] E. J. Hannan and B. G. Quinn, "The determination of the order of an autoregression," Journal of the Royal Statistical Society: Series B Methodological, vol. 41, no. 2, pp. 190-195, 1979.

[27] H. Bozdogan, "Model selection and Akaike's Information Criterion (AIC): the general theory and its analytical extensions," Psychometrika, vol. 52, no. 3, pp. 345-370, 1987. 